\title{
Marginal Trade-Offs for Improved Agro-Ecological Efficiency Using Data Envelopment Analysis
}

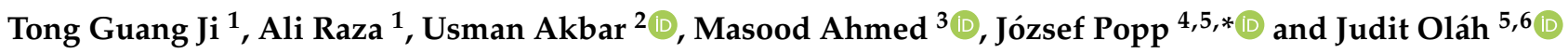 \\ 1 College of Economics and Management, Northeast Forestry University, Harbin 150040, China; \\ tonggj63@126.com (T.G.J.); ali.harbin@yahoo.com (A.R.) \\ 2 School of Economics and Management, Yanshan University, Qinhuangdao 066004, China; \\ usman.akbar@outlook.com \\ 3 Department of Public Administration, Faculty of Management Sciences, University of Kotli, Azad Jammu and \\ Kashmir, Kotli 11100, Pakistan; mrmasoodamalik@gmail.com \\ 4 Faculty of Economics and Social Sciences, Szent István University, 2100 Gödöllő, Hungary \\ 5 TRADE Research Entity, North-West University, Vanderbijlpark 1900, South Africa \\ 6 Faculty of Economics and Business, University of Debrecen, 4032 Debrecen, Hungary; \\ olah.judit@econ.unideb.hu \\ * Correspondence: popp.jozsef@uni-mate.hu; Tel.: +36-30-297-3163
}

check for updates

Citation: Ji, T.G.; Raza, A.; Akbar, U.; Ahmed, M.; Popp, J.; Oláh, J. Marginal Trade-Offs for Improved Agro-Ecological Efficiency Using Data Envelopment Analysis. Agronomy 2021, 11, 365. https:// doi.org/10.3390/agronomy11020365

Academic Editor: Thomas Bournaris

Received: 4 January 2021

Accepted: 10 February 2021

Published: 18 February 2021

Publisher's Note: MDPI stays neutral with regard to jurisdictional claims in published maps and institutional affiliations.

Copyright: (c) 2021 by the authors. Licensee MDPI, Basel, Switzerland. This article is an open access article distributed under the terms and conditions of the Creative Commons Attribution (CC BY) license (https:/ / creativecommons.org/licenses/by/ $4.0 /)$.

\begin{abstract}
Today's agricultural management decisions impact food security and sustainable ecosystems, even when operating with back-to-basic operations. In such endeavors, policymakers usually need a quantitative tool, such as trade-offs margins, to effectively adjust resource consumption or production. This paper applies the weighted slack-based measurement (SBM-DEA) program to 136 developing countries' agricultural performance. First, it finds the current agricultural efficiency and then makes marginal trade-offs on desirable-output variables (such as crop yield and forest area) to see the effective changes in undesirable-output (such as methane and nitrous oxide emissions). The results show that choosing effective marginal trade-offs does not deteriorate the relative efficiency of the decision-making units (DMUs) below the efficient frontier line. Thus, such a method enables the decision-makers to determine the best marginal trade-off points to reach the optimal efficiencies and decide which output factor needs special brainstorming to design effective policy.
\end{abstract}

Keywords: data envelopment analysis; ecological efficiency; marginal rate; trade-offs

\section{Introduction}

Agricultural efficiency makes an important contribution to the country's economy [1]. Efficiency improvements become crucial with the involvement of indirect variables [2], such as forest area, and basic agricultural emissions (even when considering machineless agricultural operations). That is, if we separate the $\mathrm{CO}_{2}$ emissions since they are connected to so many other things (i.e., machines), agricultural operations still also accompany the methane and nitrous oxide emissions as an indirect cost [3]. Therefore, resource consumption (undesirable) and production (desirable) are the key activities reflecting economic progress. Massive agricultural activity has become a potential contributor to ecological efficiency as it is directly proportional to the forest area and cultivating emissions (global carbon budget 2016 [4]). If it is not carefully planned, it can cause severe environmental problems. Therefore, to achieve higher agricultural efficiency, indirect variables must also be taken into account for real operational evaluation.

Trade-off techniques have been found widely explained theoretically, and their importance has been increased in the agricultural system to foresee the outcomes [5]. For example, a recent study by Ndiaye et al. (2019) [6] uses trade-offs between sorghum and agronomic performance stability. Kanter et al. (2018) [7] evaluates the trade-offs for sustainable developments considering agricultural systems. Conventionally, the trade-off is such an adjustment which gives the maximum output, and to see its effect on other variables 
when performed on one variable [8]. Akbar et al. [9] presented the conference paper, in which the trade-off has been performed using statistical techniques.

Moreover, finding an effective mathematical program that can be used to calculate the trade-offs is another difficult task. Data envelopment analysis (DEA) is one of the methods that can help perform marginal trade-offs. Mirzaei et al. [10] have used DEA to calculate the marginal rates of substitution to achieve optimal hydroelectric power plants optimal efficiencies. Khoshandam et al. [11] use trade-off balanced to nondiscretionary (ND) factors. Similarly, Atici and Podinovski [12] use DEA to assess the efficiency of the different agricultural units with production trade-offs. Asmild et al. [13] calculate trade-off margins with the elasticity of substitution. In their study, the new thing is that it can take negative and positive values both.

In this study, our analysis contributes in two ways. First, it enables us to bring improvement in the undesirable outputs by performing trade-offs among the desirable outputs. This means we do not have to reduce the inputs (i.e., resources like land and manpower, etc.). Second, it enables us to design better trade-off margins. Such margins do not disturb the relative efficiency of the DMUs below the frontier line but bring upon optimal efficiencies among the weakly efficient DMUs. By this, we mean that the DMUs may have appeared efficient (i.e., on the efficient frontier), but due to slacks in an undesirable output, they are considered weakly efficient. Hence, their efficiency can be further improved to the optimal level with the help of marginal trade-offs.

This study first uses a weighted slack-based measurement (SBM) model to calculate 136 developing countries' efficiency. Then, it calculates the margins, by using the method proposed by Krivonozhko et al. [14], with which the trade-offs can be performed. In the second part (the marginal trade-offs), we consider that the inputs which are at the bottleneck and cannot be decreased or increased further (by the operational manager). Hence, the marginal trade-offs are to be considered only among desirable-outputs to improve the efficiency of undesirable-outputs. The rest of the paper is designed as follows; the next section thoroughly discusses the literature on trade-off methods and its computation with DEA. Section 3 puts forward the applicable model of SBM-DEA and marginal trade-offs, followed by an example. Section 4 discusses the measuring variables and analyzes the results. The conclusion is written in Section 5.

\section{Literature}

Agriculture industry plays an important role in the socioeconomic freedom (a tool to boost financial activities) and environmental sustainability of the developing countries $[15,16]$. If we use trade-offs as a method to cope with the optimal efficiency difficulties, the definition of trade-offs must be clear. There is a lot of research variations due to the perceived definition of quantitative trade-off methods. Some use trade-offs as a tool to find an effective balance between efficiency-related variables, for example, see the recent study of Akbar el al. [9], while others consider eliminating the trade-offs for sustainable and efficient performance, for example, Gružauskas et al. [17] and Shahbazpour and Seidel [18]. Our work adheres to the previous definition of trade-off, but to find the quantitative margins with which the trade-offs should be performed is rather challenging and new to the literature.

On the contrary, the method selection of trade-offs is also crucial, because it depends on the operational plans and gains. Therefore, we divide our literature in the following two parts. The first part discusses the recent developments in the agricultural efficiency specifically concerning emissions, which not only portrays its importance but also validates our contribution in the existing literature of the agriculture energy efficiency. The second part assesses the best fit of DEA to perform the quantitative trade-offs.

\subsection{Developments in the Agro-Ecological Efficiency}

Immense literature with considerable effective outcomes has been found for the ecological performance in agricultural operations. The most recent work of Pratt et al. 
(2020) [19] researched the opportunities where geosphere can support the agricultural system across four key challenges. One of their four findings is to mitigate the emissions of carbon dioxide $\mathrm{CO}_{2}$, methane, and nitrous oxide $\mathrm{N}_{2} \mathrm{O}$. An interesting fact indicated is that the increasing geological inputs could increase footprints in an agricultural system. Akbar et al. (2020) [20] work on the reduction of the $\mathrm{CO}_{2}$ up to the sustainable level in an agro-ecological efficiency. They find the important factors affecting agro-ecological growth, such as planting structure, value-added per capita, and scale management etcetera. Bosco et al. [21] focus on the emissions from agriculture cultivation during seasonal crop rotations. They perform trade-offs between the greenhouse gases and the crop productivity within the three cropping systems (GHG).

Most importantly, they show that the organic conversion system does not really contribute to GHG mitigation during crop rotations. Shen et al. [22] work on the methane and nitrous oxide emissions from crops and animals of 69 municipalities. The results show that the direct $\mathrm{N}_{2} \mathrm{O}$ emissions are larger than indirect emissions, and the maximum $\mathrm{N}_{2} \mathrm{O}$ emissions were found to be from synthetic fertilizers. Same is the work by Yue et al. [23] and Tang et al. [24] but considering China's long-term fertilizer management. On the other hand, Audet et al. [25] present that important sources of $\mathrm{N}_{2} \mathrm{O}$ emissions include forest streams, which is equivalent to $25 \%$ of the agricultural $\mathrm{N}_{2} \mathrm{O}$ emissions in Sweden. Wysocka et al. [26] statistically analyze the $\mathrm{N}_{2} \mathrm{O}$ and methane emissions from regional agriculture and suggest that best management practice can reduce the burden of environmental emission which may enhance the profitability. In their later work [27], they found that farmers may be asked to find different niches which are profitable and less environmentally troublesome (including GHG). There is a huge pile of literature as we move backward in the literature, however, the work of Zanist et al. [28] differs in a sense that they spot the indirect factors of $\mathrm{N}_{2} \mathrm{O}$ emissions from headwater streams and the drain fields of agriculture.

\subsection{Quantitative Trade-Offs with Data Envelopment Analysis}

A plethora of data envelopment analysis (DEA) studies is found in the decisionmaking units (DMUs). Like many other sectors (banks, hotels, hospitals, and production firms etc.), it has been widely used in the agricultural efficiencies. However, the DEA with trade-off application is rather scarce, and there is further room available for improvement and techniques. There are several DEA studies which contributed to the concept of quantitative trade-offs. Most of them used trade-off for a single measure, i.e., only among two variables, when a change is brought to one variable to see another variable's effect. Rosen et al. [29] solved the balance between two variables in DEA on the effective boundary. Sueyoshi and Goto [30] used the production method to calculate the trade-off effect, in which a set of variables weighed between them. They use nondiscretionary factors to expand the work of substitution rates. Research by Huang et al. [31] proposed a general method, which changed the rate of change from output to input along the production set's efficient boundary. Emrouznejad and Yang (2018) [32] revalidated the work of Cooper et al. [33], modified the basic additive DEA model, and used the slack of the result to design an effective trade-off and marginal rate of substitution. Chang et al. [34] also used trade-offs to evaluate the environmental efficiency of transportation. They found better performance considering the greenhouse gases emissions. Watto and Mugera [35] used a trade-off model to estimate the effective use of groundwater. Again, the slacks were used. It turns out that water buyers are not as efficient as pipeline owners.

As mentioned earlier, the above-mentioned work considered the trade-offs among the two variables only. However, our work is related to multivariable trade-off. In our further literature research, there is not much that deals with multiple variables except the few to the best of our knowledge. The work of Miller et al. (2019) [36], tailed by the findings of Mirzaei et al. [10], used elasticity of substitution between input variables. Their paper provide derivatives and parsimonious methods for estimation using DEA. Akbar et al. [37] define the further types of trade-offs among multiple factors and the way to improve the trade-offs. This was followed by their advanced work of identifying the trade-offs using 
the DEA programs [9] and the statistical methods of performing multivariable trade-offs, conceptually [9]. Considering the work of Miller et al. (2019), we can use support surface bonding with effective boundary points to define different trade-off margins. Such margins do not lose the relative efficiency of the unimproved DMU, but rather promote the weakly efficient DMUs to the best efficiency point. This paper uses the same methodology on rather complex multiple variables with a large amount of data. However, it should be noted that the piecewise linear boundary in DEA technology is not clear in some cases, and the marginal trade-offs of one or more variables can only use a smaller substitution.

\section{Model}

In this section, we first explain a little the basic model of the slack-based measurement (SBM), then the weighted SBM model is described, which is mainly used for the efficiency measurement of the decision-making units (DMUs), i.e., countries in our case. Next, we explain the trade-off program followed by the marginal trade-offs.

\subsection{Slack Based Measurement of DEA}

We present here the SBM model to be used with undesirable outputs. The basic model was presented by Ton (2001) [38]. It has two properties, and one is that its unit of the measure remains the same, and another is that it has a monotonous decrease. The following basic program is developed by Tone.

$$
\rho=\left(\frac{1}{m} \sum_{i=1}^{m} \frac{x_{i o}-s_{i}^{-}}{x_{i o}}\right)\left(\frac{1}{s} \sum_{r=1}^{s} \frac{y_{r o}+s_{r}^{+}}{y_{r o}}\right)^{-1} .
$$

The ratio $\left(x_{i o}-s_{i}^{-}\right) / x_{i o}$ gauges the virtual reduction rate of the input variable $i$; therefore, $(1 / m) \sum\left(x_{i o}-s_{i}^{-}\right) / x_{i o}$ corresponds to the reduction rate of inputs of the mean proportion. Similarly, the term $\left(y_{r o}-s_{r}^{+}\right) / y_{r o}$ evaluates the proportional expansion rate of the output $r$ and $(1 / s) \sum\left(y_{r o}+s_{r}^{+}\right) / y_{r o}$ is the mean proportional rate of output. Further, the inputs and outputs can be assigned weights as follows:

$$
\rho=\frac{1-\frac{1}{m} \sum_{i=1}^{m} w_{i}^{-} s_{i}^{-} / x_{i o}}{1+\frac{1}{s} \sum_{r=1}^{s} w_{r}^{+} s_{r}^{+} / y_{r o}}
$$

where:

$\rho=$ efficiency;

$x_{i o}=$ input variable of unit $i$;

$y_{r o}=$ output variable with $r$;

$s^{-}=$excesses in inputs;

$s^{+}=$shortages in outputs; and

$r=$ growth rate.

This choice of weights with $\sum_{i=1}^{m} w_{i}^{-}=m$ and $\sum_{r=1}^{s} w_{r}^{+}=s$ reflect the importance of the input $i$ output $r$, them being proportional to its average amplitude. Generally, DEA allows more production as outputs with taking relatively fewer inputs, but the further expansion in the SBM program includes the undesirable outputs. The evaluation of the undesirable outputs is different since it also indicates an excessive amount indicating deficiencies. Seiford and Zhu [39] presented the original DEA model in 2002 and used desirable and undesirable output methods. Later, the trade-off (relaxation values) based calculations were modified by Cooper et al. [40]. This method solves the environmental inefficiency by considering undesirable output (such as GHG). One method is to convert the undesired output values to the desired values, but this may result in a misrepresentation of the effective boundary and, therefore, result in a different efficiency score. We, therefore, adopt the undesirable values for our calculations. 
Suppose that the $n$ DMUs, each having three factors, are input $x$, desirable output $y^{g}$, and undesirable output $y^{b}$, represented by three vectors $x \in R^{m}, y^{g} \in R^{s_{1}}$, and $y^{b} \in R^{s_{2}}$, respectively. The matrices $X, Y^{g}$, and $Y^{b}$ are defined as follows:

$$
\begin{aligned}
& X=\left[\begin{array}{lll}
x_{1} & \cdots & x_{n}
\end{array}\right] \in R^{m \times n}, \\
& Y^{g}=\left[\begin{array}{lll}
y_{1}^{g} & \cdots & y_{n}^{g}
\end{array}\right] \in R^{n \times s_{1}}, \\
& Y^{b}=\left[\begin{array}{lll}
y_{1}^{b} & \cdots & y_{n}^{b}
\end{array}\right] \in R^{n \times s_{2}} .
\end{aligned}
$$

Let us assume that $X>0, Y^{g}>0$, and $Y^{b}>0$, then the production possibility set as:

$$
P=\left\{\left(x, y^{g}, y^{b}\right) \mid x \geq X \delta, y^{g} \leq Y^{g} \delta, y^{b} \geq Y^{b} \delta, \delta \geq 0\right\}
$$

As per the definition of the SBM-undesirable output model, a $D M U_{0}\left(x_{0}, y_{0}^{g}, y_{0}^{b}\right)$ is efficient in the presence of an undesirable output if there is no vector $\left(x, y^{g}, y^{b}\right) \in P$, such that $x_{0} \geq x, y_{0}^{g} \leq y^{g}, y_{0}^{b} \geq y^{b}$. Following this definition, in the case of one input, one good output, and one bad output, the modified SBM of William Cooper et al. is as follows:

$$
\rho^{*}=\min \frac{1-\frac{1}{m} \sum_{i=1}^{m} \frac{s_{i}^{-}}{x_{i o}}}{1+\frac{1}{s_{1}+s_{2}}\left(\sum_{r=1}^{s_{1}} \frac{s_{r}^{g}}{y_{r o}^{g}}+\sum_{r=1}^{s_{2}} \frac{s_{r}^{b}}{y_{r o}^{b}}\right)} .
$$

Subjected to

$$
\begin{gathered}
x_{o}=X \delta+s^{-}, \\
y_{o}^{b}=Y^{b} \delta+s^{b}, \\
y_{0}^{g}=Y^{g} \delta-s^{g}, \\
\therefore s^{-}, s^{g}, s^{b}, \delta \geq 0 .
\end{gathered}
$$

The function is strictly decreasing when $s_{i}^{-}(\forall i), s_{r}^{g}(\forall r)$ and $s_{r}^{b}(\forall r)$, and the objective value must be satisfied with the condition $0<\rho^{*} \leq 1$. The vector $s^{-} \in R^{m}$ indicates the excesses in the inputs and $s^{b} \in R^{s_{2}}$ indicates to the excesses in undesirable outputs, while $s^{g} \in R^{s_{1}}$ represents shortages in desirable outputs. In the presence of the undesired output, if $D M U_{o}$ (a decision-making unit) has optimal efficiency $\rho^{*}=1$, or we can say if the slacks are equal to zero, i.e., $s^{-}, s^{g}, s^{b}=0$, then the $D M U_{\mathrm{o}}$ is called efficient. If the $D M U_{\mathrm{o}}$ is inefficienct or if it has low efficiency, i.e., $\rho^{*}<1$, then it can be improved by reducing excessive inputs and excessive undesirable outputs or increasing the desirable output. The function $\rho^{*}$ is a decreasing function, where $s_{i}^{-} / x_{i o}$ and $s_{r /}^{b} y_{r o}^{b}$ are bounded by 1 , whereas $s_{r}^{g} / y_{r o}^{g}$ is unbounded.

Now, as this paper follows, the above program (7) can be assigned with weights. The weighted ratio of desirable to undesirable output can also be implemented on the SBM-undesirable output model.

$$
\rho^{*}=\min \frac{1-\frac{1}{m} \sum_{i=1}^{m} \frac{w_{i}^{-} s_{i}^{-}}{x_{i o}}}{1+\frac{1}{s_{1}+s_{2}}\left(\sum_{r=1}^{s_{1}} \frac{w w_{r}^{g} S_{r}^{g}}{y_{r o}^{g}}+\sum_{r=1}^{s_{2}} \frac{w_{r}^{b} s_{r}^{b}}{y_{r o}^{b}}\right)},
$$

where $w_{i}^{-}, w_{r}^{g}$, and $w_{r}^{b}$ represent the weights to the input $i$, desirable outputs $r$, and undesirable outputs $r$, respectively. Additionally, $\sum_{i=1}^{m} w_{i}^{-}=m, w_{i}^{-} \geq 0(\forall i), \sum_{r=1}^{s_{1}} w_{r}^{g}+$ $\sum_{r=1}^{s_{2}} w_{r}^{b}=s_{1}+s_{2}, w_{r}^{g} \geq 0(\forall r)$, and $w_{r}^{b} \geq 0(\forall r)$. 


\subsection{Model for Trade-Off Balances}

Mathematically, the marginal rates (MR) are used to calculate the margins with which trade-offs can be performed. In the multidimensional input and output space, the DEA production possibility set can evaluate agricultural performance without losing mathematical objectivity. By considering a general process, the marginal substitution rate is used, in which the input vector $x=x_{1}, \ldots, x_{m} \in R_{+}^{m}$. is consumed by the output vector $y=y_{1}, \ldots, y_{s} \in R_{+}^{s}$. The group of $n \mathrm{DMU}_{j}: j=1, \ldots, n$ is represented by trade-off vectors $z_{j}=\left(x_{j}, y_{j}\right)^{t}$, where $x_{j}=x_{1 j}, \ldots, x_{m j}$ and $y_{j}=y_{1 j}, \ldots, y_{s j}$ are positive vectors and are above zero.

Suppose that the efficient boundary is $F(x, y)=0$, we can assume that

$$
\frac{\delta F(x, y)}{\delta x_{i}}<0 i=1, \ldots, m \frac{\delta F(x, y)}{\delta y_{r}}<0 r=1, \ldots, s
$$

Assuming that $(x, y)$ is differentiable. Let $z_{0}=x_{0}, y_{0}$ be the efficient frontier; i.e., $F\left(z_{0}\right)=F\left(x_{0}, y_{0}=0\right)$. By definition, the trade-off $\tau$ at the efficient boundary is as follows:

$$
\begin{aligned}
& \tau_{j k}^{+}\left(z_{0}\right)=\left(\frac{\delta z_{j o}}{\delta z_{k o}}\right)_{z_{0}^{+}}=\lim _{h \rightarrow 0^{+}} \frac{f_{j}\left(z_{10}, \ldots, z_{k o}+h, \ldots, z_{m+s o}\right)}{h} \\
& \tau_{j k}^{-}\left(z_{0}\right)=\left(\frac{\delta z_{j o}}{\delta z_{k o}}\right)_{z_{0}^{-}}=\lim _{h \rightarrow 0^{-}} \frac{f_{j}\left(z_{10}, \ldots, z_{k o}+h, \ldots, z_{m+s o}\right)}{h}
\end{aligned}
$$

Asmild et al. [13] gave a program for calculating the trade-off from $j$ to $k$ as mentioned below:

$$
\tau_{j k}^{+}\left(z_{0}\right)=\frac{z_{j o}^{*}-z_{j o}}{h}
$$

where $z_{j o}^{*}$ is the optimal, efficient point, $h$ is a positive number, and it is the solution to the following linear program:

$$
\begin{gathered}
\max z_{j o}^{*} \\
\text { s.t. } \sum_{t=1}^{n} \delta_{t} z_{l t} \geq z_{l o} \quad l \neq j, k \\
\sum_{t=1}^{n} \delta_{t} z_{j t} \geq z_{j o}^{*} \\
\sum_{t=1}^{n} \delta_{t} z_{k t} \geq z_{k o}+h \\
\sum_{t=1}^{n} \delta_{t}=1 \\
\delta_{t} \geq 1
\end{gathered}
$$

\subsection{Trade-Offs with Desirable and Undesirable Outputs}

Assume that managers only focus on the agricultural system's output without considering explicit inputs (that is, inputs have been regarded as bottlenecks and cannot be reduced). We considered a nonmachine agricultural energy system to make effective trade-offs in need and undesired output. The procedure described below is the same as that designed by Mirzaei et al. [10].

Suppose there are $n D M U_{j}: j=1, \ldots, n$, with two good output vectors $y_{a j}=$ $\left(y_{1 a j}, \ldots, y_{s a j}\right) \geq 0, y_{b j}=\left(y_{1 b j}, \ldots, y_{s b j}\right) \geq 0$, and one bad output vector $z_{j}=\left(z_{1 j}, \ldots, z_{p j}\right)$ $\geq 0$. By following Shepherd's [41] assumption, the following linear production technology can be used to solve bad (undesirable) output in the transportation system without the explicit inputs.

$$
T_{\mathrm{WI}}=\left\{\left(y_{a}, y_{b}, z\right): \sum_{j=1}^{n} \delta_{j} y_{a j} \geq y_{a}, \sum_{j=1}^{n} \delta_{j} y_{b j} \geq y_{b}, \sum_{j=1}^{n} \delta_{j} z_{j} \geq z, \sum_{j=1}^{n} \delta_{j}=\theta, 0 \leq \theta \leq 1, \delta_{j} \geq 0, \forall j\right\} .
$$


The following linear program using country " $\mathrm{o}$ " can be used. min $\theta^{*}$

$$
\begin{gathered}
\text { s.t. } \sum_{j=1}^{n} \delta_{j} y_{r a j} \geq y_{r a o}, \quad r=1, \ldots, s_{a} \\
\sum_{j=1}^{n} \delta_{j} y_{r b j} \geq y_{r b o}, \quad r=1, \ldots, s_{b} \\
\sum_{j=1}^{n} \delta_{j} z_{p j} \geq \theta^{*} z_{p o}, \quad p=1, \ldots, P \\
\sum_{j=1}^{n} \delta_{j}=\theta \\
0 \leq \theta \leq 1 ., \delta_{j} \geq 0, j=1, \ldots, n .
\end{gathered}
$$

Consider that the bad output is from group N, and sound output belongs to group M. First, we have to choose a small number $h$ (positive number) to solve the linear program problem at the second step.

$$
\begin{aligned}
& \max \sum_{p \in M} d_{p}^{1} z_{p}^{+} \text {s.t. } \sum_{j=1}^{n} \delta_{j} y_{r a j} \geq y_{r a o}, r=1, \ldots, s_{a}, r \nexists N \\
& \sum_{j=1}^{n} \delta_{j} y_{\text {raj }} \geq y_{\text {rao }}+h, r \exists N \\
& \sum_{j=1}^{n} \delta_{j} y_{r b j} \geq y_{r b o}, r=1, \ldots, s_{b}, r \nexists N \\
& \sum_{j=1}^{n} \delta_{j} y_{r b j} \geq y_{r b o}+h, r \exists N \\
& \sum_{j=1}^{n} \delta_{j} z_{p j} \geq \theta^{*} z_{p o}, p=1, \ldots, P, p \nexists M \\
& \sum_{j=1}^{n} \delta_{j} z_{p j} \geq z_{p}^{+}, p \exists M \\
& \sum_{j=1}^{n} \delta_{j}=\theta \\
& 0 \leq \theta \leq 1, \delta_{\mathrm{j}}, \mathrm{z}_{\mathrm{p}}^{+} \geq 0 \\
& \max \sum_{\mathrm{p} \in \mathrm{M}} \mathrm{d}_{\mathrm{p}}^{1} \mathrm{z}_{\mathrm{p}}^{+} \\
& \text {s.t. } \sum_{j=1}^{n} \delta_{j} y_{\text {raj }} \geq y_{\text {rao }}, r=1, \ldots, s_{a}, r \nexists N \\
& \sum_{j=1}^{n} \delta_{j} y_{\text {raj }} \geq y_{\text {rao }}+h, r \exists N \\
& \sum_{\mathrm{j}=1}^{\mathrm{n}} \delta_{\mathrm{j}} \mathrm{y}_{\mathrm{rbj}} \geq \mathrm{y}_{\mathrm{rbo}}, \mathrm{r}=1, \ldots, \mathrm{s}_{\mathrm{b}}, \mathrm{r} \nexists \mathrm{N} \\
& \sum_{j=1}^{n} \delta_{j} y_{r b j} \geq y_{r b o}+h, r \exists N \\
& \sum_{j=1}^{n} \delta_{j} z_{p j} \geq \theta^{*} z_{p o}, p=1, \ldots, P, p \nexists M \\
& \sum_{j=1}^{n} \delta_{j} z_{p j} \geq z_{p}^{+}, p \exists M \\
& \sum_{j=1}^{n} \delta_{j}=\theta \\
& 0 \leq \theta \leq 1, \delta_{\mathrm{j}}, \mathrm{z}_{\mathrm{p}}^{+} \geq 0
\end{aligned}
$$


We can calculate the positive and negative trade-off rate by simply replacing $-h$ with $h$ as shown below.

$$
\begin{gathered}
\tau_{p k}^{+}\left(y_{a o}, y_{b o}, z_{o}\right)=\frac{z_{p}^{+}-z_{p o}}{h} \\
\tau_{p k}^{-}\left(y_{a o}, y_{b o}, z_{o}\right)=\frac{z_{p}^{-}-z_{p o}}{h} \\
\forall p \exists M, k \exists N
\end{gathered}
$$

\subsection{Trade-Off Rates}

It is assumed that there are objective conditions for basic trade-offs, so when the specific factor increases or even decreases by a small amount, it will determine the extreme change of the specific factor of the output vector. The below program enables us to solve the trade-offs

$$
\begin{gathered}
\max z_{k o}^{\prime} \\
\text { s.t. } z_{o}^{\prime} \in T \\
\text { where } z_{o}^{\prime}=\left(z_{10}, \ldots, z_{k o}+h, \ldots, z_{m+s o}\right)
\end{gathered}
$$

Notice the new vector above even though it is output or input, and it is increasing function. The above model looks for an effective boundary on which to maximize or minimize a specific variable. However, in any case, the new point is the effective boundary, which is our requirement.

\subsection{Illustrative Example}

First, the SMB model is applied to the simple dataset in Table 1 of seven decisionmaking units (DMUs). Then, we show this in Table 2, with two inputs $x_{1}$ and $x_{2}$, and one output $y_{1}$, all DMUs are efficient. Mirzaei et al. have used this example with the help of the BCC model. It shows that DMU is useful, but there still exist slacks in the bad output. This makes DMUs weakly efficient, and we want to perform trade-offs which may reduce the bad output to help in getting the optimal efficiency level.

Table 1. The data set.

\begin{tabular}{ccccccccc}
\hline & & & \multicolumn{9}{c}{ Decision-Making Units (DMUs) } & G \\
& & A & B & C & D & E & F & G \\
\hline Inputs & $x_{1}$ & 0.9 & 0.5 & 1.1 & 0.2 & 2.2 & 2.8 & 3 \\
and & $x_{2}$ & 1.63 & 1.36 & 1.55 & 2.15 & 2.04 & 1.40 & 2.04 \\
output & $y_{1}$ & 0.65 & 0.35 & 0.65 & 0.55 & 1.2 & 0.8 & 1.3 \\
\hline
\end{tabular}

Table 2. The efficiency and the slacks.

\begin{tabular}{ccccccccc}
\hline DMU & $x_{1}$ & $x_{2}$ & $y_{1}$ & $\rho^{*}$ & $s^{-}$ & $s_{g}^{+}$ & $s_{b}^{-}$ & Ref \\
\hline A & 0.9 & 1.63 & 0 & 1 & 0 & 0 & 1 & $\mathrm{~A}$ \\
B & 0.5 & 1.36 & 0.35 & 1 & 0 & 0 & 1 & $\mathrm{~A}$ \\
$\mathrm{C}$ & 1.1 & 1.55 & 0.65 & 1 & 0 & 0 & 0.7 & $\mathrm{~A}$ \\
$\mathrm{D}$ & 0.2 & 2.15 & 0.55 & 1 & 0 & 0 & 0.4 & $\mathrm{~A}$ \\
$\mathrm{E}$ & 2.2 & 2.04 & 1.2 & 1 & 0 & 0 & 0.8 & $\mathrm{~A}$ \\
$\mathrm{~F}$ & 2.8 & 1.40 & 0.8 & 1 & 0 & 0 & 0 & $\mathrm{G}$ \\
$\mathrm{G}$ & 3 & 2.04 & 1.3 & 1 & 0 & 0 & 0 & $\mathrm{G}$ \\
\hline
\end{tabular}

Consider $\mathrm{DMU}_{\mathrm{A}}$ with $z_{1}:\left(-x_{11},-x_{21}, y_{1}^{*}\right)=(-0.9,-1.63,0.65)$. The change is needed to $x_{1}$ and $x_{2}$ when $y_{1}$ is changed by 1 unit (i.e., from 0.65 to 0.75 ). The optimistic method gives a new point on the frontier facet ACE (because in $\mathrm{DMU}_{\mathrm{A}}$ the four surfaces $\mathrm{ABC}, \mathrm{ABD}, \mathrm{ADE}$, and $\mathrm{ACE}$ have a binding effect), where $\left(-x_{11},-x_{21}, y_{1}^{*}\right)=$ $(-1.136,-1.704,0.75)$, where $x_{11}$ indicates the first input of the first DMU (i.e., DMU A). The trade-offs rates $\tau_{13}^{+}=2.364$ and $\tau_{23}^{+}=0.745$ are the value change in the first and second 
input. This change occurs when a single output $y_{1}$ increases by 0.1 units. It is worth noting that $\left(-x_{11},-x_{21}, y_{1}^{*}\right)=(-1.136,-1.704,0.75)$ is the outcome of the following model:

$$
\begin{gathered}
\min z_{1}^{*}+z_{2}^{*} \\
\text { s.t. }-0.9 \delta_{1}-0.5 \delta_{2}-1.1 \delta_{3}-0.2 \delta_{4}-2.2 \delta_{5}-2.8 \delta_{6}-3 \delta_{7} \geq-z_{1}^{*} \\
-1.63 \delta_{1}-1.36 \delta_{2}-1.55 \delta_{3}-2.15 \delta_{4}-2.04 \delta_{5}-1.4 \delta_{6}-2.04 \delta_{7} \geq-z_{2}^{*} \\
0.65 \delta_{1}-0.35 \delta_{2}-0.65 \delta_{3}-0.55 \delta_{4}-1.2 \delta_{5}-0.8 \delta_{6}-1.3 \delta_{7} \geq 0.65+0.1 \\
\delta_{1}+\delta_{2}+\delta_{3}+\delta_{4}+\delta_{5}+\delta_{6}+\delta_{7}=1 \\
\delta_{1}, \delta_{2}, \delta_{3}, \delta_{4}, \delta_{5}, \delta_{6}, \delta_{7} \geq 0
\end{gathered}
$$

Let all points (i.e., $\mathrm{ABC}, \mathrm{ABD}$, and $\mathrm{ADE}$ ) on the boundary to calculate a new trade-off rate. Changing in the direction would give a new point on a new efficiency frontier. For example, the points $\left(-x_{11},-x_{21}, y_{1}^{*}\right)=(-0.815,-2.116,0.75)$ are an efficient point of $\mathrm{ADE}$, with trade-offs $\tau_{13}^{-}=-0.8462$ and $\tau_{23}^{-}=-4.8615$. In $\mathrm{DMU}_{\mathrm{A}}$, the two trade-off ratios, $\tau_{13}^{+}$and $\tau_{23}^{+}$indicate that if we increase $y_{1}$ from 0.65 to 0.75 (see Table 1 ), and changes are brought to the two inputs (i.e., changed to 1.1364 and 1.7045), $\mathrm{DMU}_{\mathrm{A}}$ will still stay at the efficient boundary. While $\tau_{13}^{-}$and $\tau_{23}^{-}$mean that with $h=+0.1$, if both the inputs are changed to 0.8154 and 2.1162 , respectively, it will remain on the effective boundary. Interestingly, in terms of two different efficient aspects, these two new points have a big difference in them. The point $\left(-x_{11},-x_{21}, y_{1}^{*}\right)=(-0.815,-2.116,0.75)$ is optimal because of the following program:

$$
\begin{gathered}
\min -z_{1}^{*}+z_{2}^{*} \\
\text { s.t. }-0.9 \delta_{1}-0.5 \delta_{2}-1.1 \delta_{3}-0.2 \delta_{4}-2.2 \delta_{5}-2.8 \delta_{6}-3 \delta_{7} \geq-z_{1}^{*} \\
-1.63 \delta_{1}-1.36 \delta_{2}-1.55 \delta_{3}-2.15 \delta_{4}-2.04 \delta_{5}-1.4 \delta_{6}-2.04 \delta_{7} \geq-z_{2}^{*} \\
0.65 \delta_{1}-0.35 \delta_{2}-0.65 \delta_{3}-0.55 \delta_{4}-1.2 \delta_{5}-0.8 \delta_{6}-1.3 \delta_{7} \geq 0.65+0.1 \\
\delta_{1}+\delta_{2}+\delta_{3}+\delta_{4}+\delta_{5}+\delta_{6}+\delta_{7}=1 \\
\delta_{1}, \delta_{2}, \delta_{3}, \delta_{4}, \delta_{5}, \delta_{6}, \delta_{7} \geq 0
\end{gathered}
$$

Two trade-offs $\tau_{13}^{+}, \tau_{23}^{+}$and $\tau_{13}^{-}, \tau_{23}^{-}$are calculated (i.e., marginal rate of substitution to throughput $\mathrm{N}$ from group $\mathrm{M}$ ) at an efficient point $z_{0}=-x_{0}, y_{0}$. The following three-step of Mirzaei et al. has been adapted:

1. Decide on the $h$. This should be a small number for $k$ throughput.

2. Secondly, we solve the two linear program.

$$
\begin{gathered}
z_{o}^{+}+\max \sum_{l \in M} d_{l}^{(1)} z_{l o}^{*} \\
\text { s.t. } \sum_{t=1}^{n} \delta_{t} z_{t l} \geq z_{l o}^{*}, l=1.2 \ldots, m+s \\
\sum_{t=1}^{n} \delta_{t}=1 \\
z_{l o}^{*}=z_{l o}, l \nexists M, N \\
z_{k o}^{*}=z_{k o}+h, l \in N \\
\delta_{t}, z_{l o}^{*} \geq 0, \forall t, l \\
z_{o}^{-}=\max \sum_{l \in M} d_{l}^{(2)} z_{l o}^{*} \\
\text { s.t. } \sum_{t=1}^{n} \delta_{t} z_{t l} \geq z_{l o}^{*}, l=1.2 \ldots, m+s \\
\sum_{t=1}^{n} \delta_{t}=1 \\
z_{l o}^{*}=z_{l o}, l \nexists M, N \\
z_{k o}^{*}=z_{k o}+h, l \in N \\
\delta_{t}, z_{l o}^{*} \geq 0, \forall t, l
\end{gathered}
$$

Please note that $d_{l}^{(1)}$ and $d_{l}^{(2)}$ are the constant numbers, user-defined. 
3. Calculate the trade-off of negative and positive rates from the right, as follows:

$$
\begin{aligned}
& \tau_{j k}^{+}\left(z_{o}\right)=\frac{z_{j o}^{+}-z_{j o}}{h} \\
& \tau_{j k}^{-}\left(z_{o}\right)=\frac{z_{j o}^{-}-z_{j o}}{h} \\
& \forall j \in M, k \in N
\end{aligned}
$$

Trade-offs can be calculated by replacing $-h$ with $h$. Because both the programs (26) and (25) make projections on the frontier points. Moreover, different trade-offs will result in other weighted vectors $d$. The weighted vector $d$ is user-defined; it determines the efficient surface direction.

\section{Analysis and Discussion}

\subsection{Efficiency Measuring Variables}

Given developing countries, it can be observed from Akbar et al. (2020) [42,43] that the undesirable-outputs in any operations are not separable from the desirable-outputs, i.e., crop production and forest area. Reducing undesirable-output in practice inevitably leads to a reduction in desirable-output variables. Besides, certain undesirable-outputs can have significant effects (inseparable) on specific inputs. For example, emissions of methane and nitrous oxide gases (undesirable-outputs) in agricultural operations are proportional to the forest area and agricultural land area.

To illustrate the trade-offs, we selected the data consist of 136 countries. Here, the latest data set of the year 2019 is composed of multiple outputs and input variables, see Appendix A. For the trade-offs application, only the output variables are considered because the inputs are assumed to have reached the bottleneck in both ways that it cannot be increased or decreased by the managers. Among the four indicators, two are regarded as good-output variables, and two are the bad-output variables. The total crop production in each country is considered the first good-output $y_{1}^{g}$, and the forest area is the second desirable-output $y_{2}^{g}$. It is assumed that the more the forest area a DMU has, the less the area of crop production it utilizes. Not to mention that the crop production symbolizes the soil erosion and other operational activity causing emissions. The undesirable-outputs are methane emissions $z_{1}^{b}$ and nitrous oxide emissions $z_{2}^{b}$ purely from agricultural activity (considering the nonmachine agricultural operations), which positively correlates the two good-outputs. These indicators also relate to the ecological efficiency of agricultural operations, see Table 3.

Table 3. Variables for the agro-ecological efficiency.

\begin{tabular}{cccc}
\hline & Variables & Unit of Measure & Source \\
\hline \multicolumn{1}{l}{ Inputs } & & & \\
\hline$x_{1}$ & Freshwater consumption & Billion cubic meters & World Bank \\
$x_{2}$ & Agricultural land area & Percentage of land & World Bank \\
$x_{3}$ & Fertilizer consumption & Percentage of fertilizer production & World Bank \\
$x_{4}$ & Labor & Percentage of total employment & World Bank \\
\hline Desirable Outputs & & & \\
\hline$y_{1}^{g}$ & Production & Crop production index & World Bank \\
$y_{2}^{g}$ & Forest area & Percentage of land area & World Bank \\
\hline Undesirable Output & & \\
\hline$z_{1}^{b}$ & Methane emissions & Percentage of total emissions & World Bank \\
$z_{2}^{b}$ & Nitrous oxide emissions & Percentage of total emissions & World Bank \\
\hline \multicolumn{5}{c}{} & &
\end{tabular}




\subsection{Results and Discussion}

The data set was evaluated for the countries' efficiencies using a program (12), and then we re-evaluated it using the model program (18). The data was taken as a whole without conversion for better analysis. The relative efficiency obtained using the model (12) is listed in Table 4 . Countries with efficiency score 1 are fully efficient. We can see that 31 out of 136 countries have appeared efficient (i.e., when DMU =1).

Table 4. The efficiency results of 136 decision making units "DMUs" (countries).

\begin{tabular}{|c|c|c|c|c|c|c|c|}
\hline DMUs & Eff. & DMUs & Eff. & DMUs & Eff. & DMUs & Eff. \\
\hline Afghanistan & 0.09 & Estonia & 1.00 & Eritrea & 0.18 & Zimbabwe & 0.23 \\
\hline Albania & 0.25 & Ethiopia & 0.34 & Namibia & 0.13 & Yemen & 0.11 \\
\hline Algeria & 1.00 & Fiji & 0.36 & Nepal & 0.24 & Zambia & 0.58 \\
\hline Angola & 1.00 & Finland & 1.00 & Netherlands & 0.12 & Vietnam & 0.07 \\
\hline $\begin{array}{l}\text { Antigua, } \\
\text { Barbuda }\end{array}$ & 1.00 & France & 0.16 & Nicaragua & 0.21 & Ukraine & 1.00 \\
\hline Argentina & 1.00 & Gabon & 1.00 & Niger & 1.00 & UAE & 0.18 \\
\hline Armenia & 0.20 & Gambia & 0.58 & Nigeria & 0.15 & UK & 0.12 \\
\hline Australia & 0.20 & Georgia & 0.12 & Norway & 0.25 & Uruguay & 0.14 \\
\hline Austria & 0.22 & Germany & 0.18 & Oman & 0.00 & Uzbekistan & 0.07 \\
\hline Azerbaijan & 0.33 & Ghana & 0.45 & Pakistan & 0.02 & Venezuela & 0.16 \\
\hline $\begin{array}{l}\text { Bahamas, } \\
\text { The }\end{array}$ & 1.00 & Greece & 0.14 & Panama & 0.38 & Malta & 0.08 \\
\hline Bangladesh & 0.06 & Guatemala & 0.11 & $\begin{array}{c}\text { Papua New } \\
\text { Guinea }\end{array}$ & 1.00 & Mauritius & 0.10 \\
\hline Barbados & 0.31 & Guinea & 0.39 & Paraguay & 0.24 & Mexico & 0.19 \\
\hline Belarus & 0.17 & Guyana & 1.00 & Philippines & 0.13 & Moldova & 0.49 \\
\hline Belgium & 0.21 & Honduras & 0.16 & Poland & 0.14 & Mongolia & 0.46 \\
\hline Belize & 0.12 & Hungary & 0.15 & Portugal & 0.14 & Morocco & 0.16 \\
\hline Benin & 0.91 & Iceland & 0.01 & Romania & 0.19 & Dominica & 1.00 \\
\hline Bhutan & 1.00 & Iran & 0.15 & Rwanda & 0.51 & Domin. Rep. & 0.36 \\
\hline Bolivia & 0.63 & Iraq & 0.11 & Saudi Arabia & 0.01 & Ecuador & 0.09 \\
\hline $\begin{array}{c}\text { Bosnia, } \\
\text { Herzegovina }\end{array}$ & 0.17 & Israel & 0.22 & Senegal & 0.58 & Egypt & 0.00 \\
\hline Botswana & 0.16 & Italy & 0.17 & Slovak & 0.30 & Mozambique & 1.00 \\
\hline $\begin{array}{c}\text { Brunei } \\
\text { Darussalam }\end{array}$ & 1.00 & Jamaica & 0.27 & Slovenia & 0.19 & El Salvador & 0.09 \\
\hline Bulgaria & 0.22 & Japan & 1.00 & South Africa & 0.18 & $\begin{array}{l}\text { Trinidad, } \\
\text { Tobago }\end{array}$ & 1.00 \\
\hline Burkina Faso & 0.35 & Jordan & 0.07 & Spain & 0.17 & Tunisia & 0.24 \\
\hline Burundi & 1.00 & Kazakhstan & 1.00 & Sri Lanka & 0.18 & Turkey & 0.13 \\
\hline Cambodia & 1.00 & Kenya & 0.17 & $\begin{array}{l}\text { St. Kitts, } \\
\text { Nevis }\end{array}$ & 1.00 & Uganda & 0.11 \\
\hline Cameroon & 1.00 & Korea & 0.16 & St. Lucia & 0.28 & Madagascar & 0.21 \\
\hline $\begin{array}{l}\text { Central } \\
\text { African }\end{array}$ & 1.00 & Kuwait & 1.00 & Suriname & 1.00 & Malawi & 0.54 \\
\hline Chile & 0.10 & Kyrgyz & 0.05 & Sweden & 1.00 & Maldives & 1.00 \\
\hline Congo, Dem. & 1.00 & Latvia & 0.55 & Switzerland & 0.16 & Mali & 0.13 \\
\hline Congo, Rep. & 1.00 & Lebanon & 0.06 & Tajikistan & 0.07 & Cuba & 0.13 \\
\hline Costa Rica & 0.08 & Lithuania & 1.00 & Tanzania & 0.62 & Cyprus & 0.17 \\
\hline Cote d'Ivoire & 0.38 & Luxembourg & 0.12 & Thailand & 0.14 & $\begin{array}{c}\text { Czech } \\
\text { Republic }\end{array}$ & 0.20 \\
\hline Croatia & 0.23 & Macedonia & 0.31 & Togo & 0.18 & Denmark & 0.11 \\
\hline
\end{tabular}

Suppose $M=\{1,2\}$ and $N=\{1\}$. We wanted to calculate the effects of the marginal trade-off (i.e., change in the undesirable-outputs, methane emissions $z_{1}^{b}$ and nitrous oxide emissions $z_{2}^{b}$ ), when applied to the first desirable-output "crop production $y_{1}^{g \text { " }}$ and then

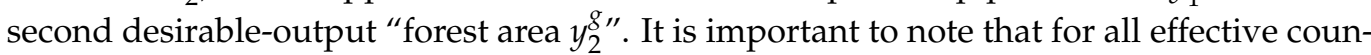
tries, $h=0.5$ and $h=-0.5$ proved to be feasible margins. This means that the increase or decrease within the marginal range (i.e., between 0.5 and -0.5) can further improve the 
macro efficiency due to the reduction in the excessive undesirable-outputs (indirect measures). Additionally, this is of course while maintaining other DMU's efficiency point on the frontier boundary, even if there is no improvement. In Appendix B, Tables A1 and A2 show the trade-off effects (of all the 136 countries) on the methane $\left(z_{1}^{b}\right)$ and nitrous oxide $\left(z_{2}^{b}\right)$ when $h=+0.5,-0.5$ and $d=(1,1)$. Here, within the text, only non-zero DMUs will be discussed, and DMUs with zero change are not mentioned (but they can be found in Appendix B).

Now, to explain our experiments concisely, we shall go step by step discussing each bad output separately in order to identify the better trade-off point. Tables $5-8$ show the results of the new value of undesirable outputs $\left(z_{1}^{b}\right.$ and $\left.z_{2}^{b}\right)$ when trade-off $h= \pm 0.5$ is applied separately on desirable-outputs $y_{1}^{g}$ and $y_{2}^{g}$. The second columns in each resultant table show the trade-off value $\left(\tau^{ \pm}\right)$with which trade-off takes place for each DMU.

Table 5. Trade-off effects on the methane $\left(z_{1}^{b}\right)$ when $h=+0.5$ and $d=(1,1)$.

\begin{tabular}{|c|c|c|c|c|c|c|c|c|c|}
\hline \multirow[b]{2}{*}{$\mathrm{DMU}_{j}$} & \multicolumn{2}{|c|}{ w.r.t $y_{1}^{g}$} & \multicolumn{2}{|c|}{ w.r.t $y_{2}^{g}$} & \multirow[b]{2}{*}{$\mathbf{D M U}_{j}$} & \multicolumn{2}{|c|}{ w.r.t. $y_{1}^{g}$} & \multicolumn{2}{|c|}{ w.r.t $y_{2}^{g}$} \\
\hline & $z_{1}^{b+}$ & $\tau_{11}^{+}$ & $z_{1}^{b+}$ & $\tau_{12}^{+}$ & & $z_{1}^{b+}$ & $\tau_{11}^{+}$ & $z_{1}^{b+}$ & $\tau_{12}^{+}$ \\
\hline Afghanistan & 11.4042 & 0.0071 & 11.3971 & 0.0000 & Latvia & 0.5898 & 0.0150 & 0.6966 & 0.1218 \\
\hline Albania & 34.3414 & 0.1826 & 34.1588 & 0.0000 & Lebanon & 25.1189 & -0.0018 & 25.1207 & 0.0000 \\
\hline Austria & 45.9628 & -0.0018 & 45.9669 & 0.0023 & Luxembourg & 30.2811 & 0.0367 & 30.2703 & 0.0259 \\
\hline Bangladesh & 62.1303 & 0.0001 & 62.1302 & 0.0000 & Macedonia & 5.9849 & 0.0147 & 5.9781 & 0.0079 \\
\hline Belarus & 43.2344 & 0.0124 & 43.1816 & -0.0404 & Madagascar & 32.1777 & -0.0019 & 32.1732 & -0.0064 \\
\hline Belgium & 43.6960 & 0.0587 & 43.6373 & 0.0000 & Malawi & 7.4308 & 0.0124 & 7.5967 & 0.1783 \\
\hline Belize & 51.0052 & 0.0000 & 51.0072 & 0.0019 & Mauritius & 13.5227 & -0.0022 & 13.5250 & 0.0000 \\
\hline Benin & 14.3868 & 0.0667 & 14.5769 & 0.2568 & Mongolia & 6.3483 & -0.5413 & 6.8895 & 0.0000 \\
\hline Bolivia & 20.7527 & -0.0007 & 20.7518 & -0.0015 & Namibia & 1.3388 & 0.2113 & 1.1275 & 0.0000 \\
\hline Bosnia and & & & & & & & & & \\
\hline $\begin{array}{l}\text { Herzegov- } \\
\text { ina }\end{array}$ & 34.9513 & 0.0122 & 34.8993 & -0.0397 & Nepal & 74.4329 & -0.0029 & 74.4375 & 0.0018 \\
\hline Bulgaria & 7.0032 & -0.0009 & 7.0042 & 0.0000 & Nicaragua & 52.3281 & 0.0040 & 52.3819 & 0.0578 \\
\hline Chile & 33.6971 & -0.0011 & 33.6982 & 0.0000 & Norway & 7.1694 & -0.0028 & 7.1756 & 0.0034 \\
\hline Costa Rica & 61.9982 & -0.0001 & 61.9984 & 0.0001 & Oman & 4.4126 & -0.0003 & 4.4128 & 0.0000 \\
\hline $\begin{array}{c}\text { Cote } \\
\text { d'Ivoire }\end{array}$ & 12.0108 & -0.0042 & 12.0177 & 0.0027 & Pakistan & 30.4262 & -0.0306 & 30.4568 & 0.0000 \\
\hline Croatia & 16.7472 & -0.0008 & 16.7480 & 0.0000 & Panama & 19.0953 & 0.0933 & 18.8226 & -0.1794 \\
\hline Cuba & 5.5215 & 0.0240 & 5.5143 & 0.0169 & Philippines & 54.0918 & -0.0010 & 54.0928 & 0.0000 \\
\hline Cyprus & 40.0264 & 0.0000 & 40.0179 & -0.0085 & Poland & 21.0054 & -0.0013 & 21.0066 & 0.0000 \\
\hline Czech Rep. & 28.4054 & -0.0013 & 28.4067 & 0.0000 & Portugal & 26.1282 & -0.0013 & 26.1295 & 0.0000 \\
\hline Ecuador & 64.6114 & -0.0010 & 64.6135 & 0.0012 & Senegal & 31.8184 & 0.0691 & 31.9967 & 0.2475 \\
\hline Egypt & 26.8410 & -0.0009 & 26.8419 & 0.0000 & Slovak Rep. & 21.7400 & 0.0160 & 21.6718 & -0.0522 \\
\hline Eritrea & 28.1564 & 0.0090 & 28.1474 & 0.0000 & Slovenia & 26.3791 & 0.0000 & 26.3819 & 0.0028 \\
\hline Fiji & 52.9460 & -0.0077 & 52.9780 & 0.0243 & Sri Lanka & 55.5878 & -0.0005 & 55.5883 & 0.0000 \\
\hline $\begin{array}{l}\text { Gambia, } \\
\text { The }\end{array}$ & 12.8958 & 0.0367 & 12.9796 & 0.1206 & St. Lucia & 31.6428 & 0.0247 & 31.5377 & -0.0804 \\
\hline Georgia & 40.2571 & 0.0000 & 40.2544 & -0.0027 & Switzerland & 57.7132 & -0.0027 & 57.7192 & 0.0033 \\
\hline Ghana & 7.1747 & 0.0142 & 7.3637 & 0.2032 & Thailand & 52.6556 & -0.0044 & 52.6708 & 0.0109 \\
\hline Greece & 32.6785 & 0.0322 & 32.5415 & -0.1048 & Turkey & 22.8057 & -0.0011 & 22.8067 & 0.0000 \\
\hline Guatemala & 47.2316 & 0.0007 & 47.2309 & 0.0000 & Uganda & 14.3203 & 0.0123 & 40.7055 & 26.3974 \\
\hline Guinea & 18.7337 & 0.0029 & 18.7488 & 0.0180 & UAE & 5.3534 & -0.0024 & 5.3531 & -0.0028 \\
\hline Honduras & 72.4080 & -0.0004 & 72.4084 & 0.0000 & Uzbekistan & 19.9724 & 0.0006 & 19.9717 & 0.0000 \\
\hline Iceland & 54.8233 & 0.0000 & 54.8234 & 0.0000 & Venezuela & 38.2000 & -0.0013 & 38.2028 & 0.0016 \\
\hline Iran & 17.4403 & -0.0077 & 17.4480 & 0.0000 & Vietnam & 52.2606 & -0.0003 & 52.2612 & 0.0003 \\
\hline Iraq & 0.0000 & 0.0000 & 5.3387 & 5.3387 & Zambia & 35.7680 & -0.0184 & 35.8049 & 0.0184 \\
\hline Jamaica & 3.9368 & -0.0025 & 3.9595 & 0.0202 & Zimbabwe & 35.3227 & 0.0079 & 35.3408 & 0.0259 \\
\hline Korea, Rep. & 33.3108 & -0.0007 & 33.3123 & 0.0008 & & & & & \\
\hline
\end{tabular}


Table 6. Trade-off effects on the methane $\left(z_{1}^{b}\right)$ when $h=-0.5$ and $d=(1,1)$.

\begin{tabular}{|c|c|c|c|c|c|c|c|c|c|}
\hline \multirow[b]{2}{*}{$\mathrm{DMU}_{\mathrm{j}}$} & \multicolumn{2}{|c|}{ w.r.t $y_{1}^{g}$} & \multicolumn{2}{|c|}{ w.r.t $y_{2}^{g}$} & \multirow[b]{2}{*}{$\mathrm{DMU}_{j}$} & \multicolumn{2}{|c|}{ w.r.t $y_{1}^{g}$} & \multicolumn{2}{|c|}{ w.r.t $y_{2}^{g}$} \\
\hline & $z_{1}^{b-}$ & $\tau_{11}^{-}$ & $z_{1}^{b-}$ & $\tau_{12}^{-}$ & & $z_{1}^{b-}$ & $\tau_{11}^{-}$ & $z_{1}^{b-}$ & $\tau_{12}^{-}$ \\
\hline Afghanistan & 11.3900 & -0.0071 & 11.3971 & 0.0000 & Luxembourg & 30.2075 & -0.0369 & 30.2180 & -0.0264 \\
\hline Albania & 33.9745 & -0.1843 & 34.1588 & 0.0000 & Macedonia & 5.9554 & -0.0148 & 5.9622 & -0.0081 \\
\hline Austria & 45.9665 & 0.0018 & 45.9624 & -0.0023 & Madagascar & 32.1815 & 0.0019 & 32.1861 & 0.0065 \\
\hline Azerbaijan & 0.0000 & 0.0000 & 0.0000 & 0.0000 & Malawi & 7.4059 & -0.0125 & 7.2361 & -0.1823 \\
\hline Bangladesh & 62.1300 & -0.0001 & 62.1302 & 0.0000 & Maldives & 0.0000 & 0.0000 & 0.0000 & 0.0000 \\
\hline Belarus & 43.2096 & -0.0124 & 43.2634 & 0.0414 & Mauritius & 13.5272 & 0.0023 & 13.5250 & 0.0000 \\
\hline Belgium & 43.5784 & -0.0589 & 43.6373 & 0.0000 & Mongolia & 7.4302 & 0.5406 & 6.8895 & 0.0000 \\
\hline Belize & 51.0052 & 0.0000 & 51.0033 & -0.0020 & Namibia & 0.9161 & -0.2115 & 1.1275 & 0.0000 \\
\hline Benin & 14.2538 & -0.0664 & 14.0673 & -0.2528 & Nepal & 74.4386 & 0.0029 & 74.4339 & -0.0018 \\
\hline Bhutan & 0.0000 & 0.0000 & 0.0000 & 0.0000 & Netherlands & 0.0000 & 0.0000 & 0.0000 & 0.0000 \\
\hline Bolivia & 20.7541 & 0.0007 & 20.7549 & 0.0015 & Nicaragua & 52.3201 & -0.0040 & 52.2650 & -0.0591 \\
\hline Bosnia and & & & & & & & & & \\
\hline $\begin{array}{l}\text { Herzegov- } \\
\text { ina }\end{array}$ & 34.9269 & -0.0122 & 34.9798 & 0.0407 & Niger & 0.0000 & 0.0000 & 0.0000 & 0.0000 \\
\hline Botswana & 0.0000 & 0.0000 & 0.0000 & 0.0000 & Norway & 7.1749 & 0.0028 & 7.1687 & -0.0035 \\
\hline Bulgaria & 7.0051 & 0.0009 & 7.0042 & 0.0000 & Oman & 4.4131 & 0.0003 & 4.4128 & 0.0000 \\
\hline Chile & 33.6993 & 0.0011 & 33.6982 & 0.0000 & Pakistan & 30.4875 & 0.0307 & 30.4568 & 0.0000 \\
\hline Costa Rica & 61.9984 & 0.0001 & 61.9981 & -0.0001 & Panama & 18.9085 & -0.0935 & 19.1822 & 0.1802 \\
\hline $\begin{array}{c}\text { Cote } \\
\text { d'Ivoire }\end{array}$ & 12.0193 & 0.0043 & 12.0124 & -0.0027 & $\begin{array}{c}\text { Papua New } \\
\text { Guinea }\end{array}$ & 0.0000 & 0.0000 & 0.0000 & 0.0000 \\
\hline Croatia & 16.7488 & 0.0008 & 16.7480 & 0.0000 & Philippines & 54.0938 & 0.0010 & 54.0928 & 0.0000 \\
\hline Cuba & 5.4733 & -0.0241 & 5.4802 & -0.0173 & Poland & 21.0079 & 0.0013 & 21.0066 & 0.0000 \\
\hline Cyprus & 40.0264 & 0.0000 & 40.0351 & 0.0087 & Portugal & 26.1308 & 0.0013 & 26.1295 & 0.0000 \\
\hline Czech Rep. & 28.4080 & 0.0013 & 28.4067 & 0.0000 & $\begin{array}{c}\text { Saudi } \\
\text { Arabia }\end{array}$ & 0.0000 & 0.0000 & 5.3588 & 5.3588 \\
\hline Ecuador & 64.6133 & 0.0010 & 64.6111 & -0.0012 & Senegal & 31.6801 & -0.0692 & 31.5000 & -0.2492 \\
\hline Egypt & 26.8428 & 0.0009 & 26.8419 & 0.0000 & Slovak Rep. & 21.7080 & -0.0160 & 21.7774 & 0.0535 \\
\hline Eritrea & 28.1384 & -0.0090 & 28.1474 & 0.0000 & Slovenia & 26.3791 & 0.0000 & 26.3763 & -0.0028 \\
\hline Fiji & 52.9614 & 0.0077 & 52.9291 & -0.0245 & $\begin{array}{l}\text { South } \\
\text { Africa }\end{array}$ & 0.0000 & 0.0000 & 0.0000 & 0.0000 \\
\hline Finland & 0.0000 & 0.0000 & 0.0000 & 0.0000 & Sri Lanka & 55.5888 & 0.0005 & 55.5883 & 0.0000 \\
\hline $\begin{array}{l}\text { Gambia, } \\
\text { The }\end{array}$ & 12.8221 & -0.0369 & 12.7357 & -0.1233 & St. Lucia & 31.5935 & -0.0246 & 31.7005 & 0.0824 \\
\hline Georgia & 40.2571 & 0.0000 & 40.2599 & 0.0028 & Suriname & 0.0000 & 0.0000 & 0.0000 & 0.0000 \\
\hline Germany & 0.0000 & 0.0000 & 0.0000 & 0.0000 & Switzerland & 57.7186 & 0.0027 & 57.7125 & -0.0034 \\
\hline Ghana & 7.1463 & -0.0142 & 6.9528 & -0.2077 & Tajikistan & 0.0000 & 0.0000 & 0.0000 & 0.0000 \\
\hline Greece & 32.6142 & -0.0321 & 32.7537 & 0.1074 & Thailand & 52.6643 & 0.0044 & 52.6490 & -0.0109 \\
\hline Guatemala & 47.2302 & -0.0007 & 47.2309 & 0.0000 & Togo & 0.0000 & 0.0000 & 0.0000 & 0.0000 \\
\hline Guinea & 18.7279 & -0.0029 & 18.7129 & -0.0179 & Turkey & 22.8078 & 0.0011 & 22.8067 & 0.0000 \\
\hline Guyana & 0.0000 & 0.0000 & 0.0000 & 0.0000 & Uganda & 14.2958 & -0.0123 & 14.3081 & 0.0000 \\
\hline Honduras & 72.4088 & 0.0004 & 72.4084 & 0.0000 & Ukraine & 0.0000 & 0.0000 & 0.0000 & 0.0000 \\
\hline Iceland & 54.8234 & 0.0000 & 54.8234 & 0.0000 & UAE & 5.3583 & 0.0025 & 5.3589 & 0.0030 \\
\hline Iran & 17.4558 & 0.0078 & 0.0000 & -17.4480 & UK & 0.0000 & 0.0000 & 0.0000 & 0.0000 \\
\hline Israel & 0.0000 & 0.0000 & 0.0000 & 0.0000 & Uzbekistan & 19.9711 & -0.0006 & 0.0000 & -19.9717 \\
\hline Jamaica & 3.9417 & 0.0025 & 3.9190 & -0.0203 & Venezuela & 38.2025 & 0.0013 & 38.1997 & -0.0016 \\
\hline Japan & 0.0000 & 0.0000 & 0.0000 & 0.0000 & Vietnam & 52.2611 & 0.0003 & 52.2606 & -0.0003 \\
\hline Korea, Rep. & 33.3122 & 0.0007 & 33.3106 & -0.0009 & $\begin{array}{l}\text { Yemen, } \\
\text { Rep. }\end{array}$ & 0.0000 & 0.0000 & 0.0000 & 0.0000 \\
\hline Kuwait & 0.0000 & 0.0000 & 0.0000 & 0.0000 & Zambia & 35.8049 & 0.0184 & 35.7679 & -0.0186 \\
\hline Latvia & 0.5596 & -0.0151 & 0.4502 & -0.1245 & Zimbabwe & 35.3069 & -0.0079 & 35.2883 & -0.0265 \\
\hline Lebanon & 25.1225 & 0.0018 & 25.1207 & 0.0000 & & & & & \\
\hline
\end{tabular}


Table 7. Trade-off effects on the nitrous oxide $\left(z_{2}^{b}\right)$ when $h=+0.5$ and $d=(1,1)$.

\begin{tabular}{|c|c|c|c|c|c|c|c|c|c|}
\hline \multirow{2}{*}{$\mathrm{DMU}_{j}$} & \multicolumn{2}{|c|}{ w.r.t $y_{1}^{g}$} & \multicolumn{2}{|c|}{ w.r.t $y_{2}^{g}$} & \multirow{2}{*}{$\mathrm{DMU}_{j}$} & \multicolumn{2}{|c|}{ w.r.t $y_{1}^{g}$} & \multicolumn{2}{|c|}{ w.r.t $y_{2}^{g}$} \\
\hline & $z_{2}^{b+}$ & $\tau_{21}^{+}$ & $z_{1}^{b+}$ & $\tau_{22}^{+}$ & & $z_{2}^{b+}$ & $\tau_{21}^{+}$ & $z_{2}^{b+}$ & $\tau_{22}^{+}$ \\
\hline Albania & 37.2237 & 0.1274 & 37.0963 & 0.0000 & Jordan & 44.6334 & 0.0005 & 44.6329 & 0.0000 \\
\hline Armenia & 16.5679 & 0.0584 & 16.5095 & 0.0000 & Korea & 42.2038 & 0.0002 & 42.1734 & -0.0302 \\
\hline Australia & 49.3246 & -0.0034 & 49.3280 & 0.0000 & Latvia & 34.2498 & 0.0686 & 34.4448 & 0.2636 \\
\hline Austria & 38.4120 & 0.0006 & 38.3310 & -0.0804 & Lebanon & 64.8009 & -0.0405 & 64.8415 & 0.0000 \\
\hline Azerbaijan & 11.0237 & -0.0073 & 11.0310 & 0.0000 & Luxembourg & 1.1464 & 0.0145 & 1.2145 & 0.0826 \\
\hline Bangladesh & 54.2884 & 0.0028 & 54.2856 & 0.0000 & Macedonia & 28.2280 & 0.0064 & 28.2486 & 0.0269 \\
\hline Barbados & 8.4088 & -0.0218 & 8.1748 & -0.2558 & Madagascar & 40.0645 & -0.0034 & 40.0531 & -0.0148 \\
\hline Belarus & 40.8051 & 0.0154 & 40.7027 & -0.0871 & Malawi & 3.1914 & 0.0796 & 3.4974 & 0.3856 \\
\hline Belize & 33.9164 & 0.0000 & 33.9593 & 0.0429 & Malta & 8.3159 & 0.0085 & 8.3075 & 0.0000 \\
\hline Bolivia & 15.4115 & 0.0005 & 15.4105 & -0.0004 & Mauritius & 38.7434 & -0.0499 & 38.7932 & 0.0000 \\
\hline Bosnia and & & & & & & & & & \\
\hline $\begin{array}{l}\text { Herzegov- } \\
\text { ina }\end{array}$ & 62.5839 & 0.0151 & 62.4832 & -0.0856 & Mexico & 27.9968 & -0.0033 & 28.0264 & 0.0264 \\
\hline Botswana & 46.6913 & 0.0157 & 46.6756 & 0.0000 & Moldova & 39.0884 & -0.0090 & 39.0082 & -0.0892 \\
\hline Bulgaria & 31.6801 & -0.0209 & 31.7010 & 0.0000 & Nepal & 43.0448 & -0.0177 & 43.0762 & 0.0137 \\
\hline Chile & 35.4870 & -0.0252 & 35.5122 & 0.0000 & Netherlands & 38.1764 & -0.0090 & 38.1854 & 0.0000 \\
\hline Costa Rica & 45.8674 & 0.0000 & 45.8621 & -0.0053 & Nicaragua & 24.3967 & 0.0258 & 24.4959 & 0.1250 \\
\hline $\begin{array}{c}\text { Cote } \\
\text { d'Ivoire }\end{array}$ & 3.4436 & -0.0260 & 3.4897 & 0.0201 & Norway & 41.9804 & 0.0009 & 41.8580 & -0.1215 \\
\hline Croatia & 26.9003 & -0.0176 & 26.9179 & 0.0000 & Oman & 26.1590 & -0.0063 & 26.1652 & 0.0000 \\
\hline Cuba & 16.9863 & 0.0094 & 17.0308 & 0.0540 & Paraguay & 31.8761 & 0.0653 & 31.7728 & -0.0381 \\
\hline Cyprus & 42.0092 & 0.0000 & 41.8203 & -0.1889 & Philippines & 56.6386 & -0.0223 & 56.6609 & 0.0000 \\
\hline Czech Rep. & 65.2515 & -0.0284 & 65.2799 & 0.0000 & Poland & 34.4932 & -0.0280 & 34.5212 & 0.0000 \\
\hline Denmark & 58.9339 & -0.0029 & 58.9369 & 0.0000 & Portugal & 38.1376 & -0.0294 & 38.1670 & 0.0000 \\
\hline Dominican & 18.4475 & 0.0403 & 18.4072 & 0.0000 & Romania & 5.6961 & 0.0154 & 5.7570 & 0.0763 \\
\hline Ecuador & 54.8178 & 0.0003 & 54.7752 & -0.0423 & Rwanda & 11.4551 & 0.0261 & 11.5463 & 0.1173 \\
\hline Egypt & 41.6727 & -0.0197 & 41.6924 & 0.0000 & $\begin{array}{c}\text { Saudi } \\
\text { Arabia }\end{array}$ & 18.3427 & -0.0295 & 18.3722 & 0.0000 \\
\hline El Salvador & 30.9759 & -0.0044 & 30.9802 & 0.0000 & Slovak Rep. & 1.8577 & 0.0198 & 1.7255 & -0.1124 \\
\hline Ethiopia & 14.4607 & -0.0664 & 24.8439 & 10.3167 & Slovenia & 35.5002 & 0.0000 & 35.5621 & 0.0619 \\
\hline Fiji & 21.7064 & 0.0134 & 21.7505 & 0.0575 & Spain & 5.7476 & -0.0139 & 5.8735 & 0.1120 \\
\hline France & 39.4901 & 0.0007 & 39.4550 & -0.0344 & Sri Lanka & 33.7308 & -0.0115 & 33.7423 & 0.0000 \\
\hline $\begin{array}{l}\text { Gambia, } \\
\text { The }\end{array}$ & 0.4388 & 0.0642 & 0.6542 & 0.2795 & St. Lucia & 1.7324 & 0.0306 & 1.5287 & -0.1732 \\
\hline Georgia & 25.5848 & 0.0000 & 25.5247 & -0.0601 & Switzerland & 41.0546 & 0.0009 & 40.9359 & -0.1179 \\
\hline Germany & 10.6484 & -0.0205 & 10.6761 & 0.0072 & Thailand & 38.9072 & -0.0267 & 38.9528 & 0.0189 \\
\hline Ghana & 4.8027 & 0.0907 & 5.1514 & 0.4394 & Tunisia & 8.8994 & 0.0388 & 8.8606 & 0.0000 \\
\hline Greece & 41.5020 & 0.0399 & 41.2363 & -0.2258 & Turkey & 48.6553 & -0.0234 & 48.6787 & 0.0000 \\
\hline Guatemala & 25.0176 & 0.0153 & 25.0023 & 0.0000 & Uganda & 0.0000 & 0.0000 & 61.1962 & 61.1962 \\
\hline Honduras & 41.7701 & -0.0093 & 41.7795 & 0.0000 & UAE & 27.0438 & -0.0374 & 26.9642 & -0.1170 \\
\hline Hungary & 88.6208 & -0.0309 & 88.6517 & 0.0000 & UK & 23.7600 & -0.0133 & 23.7733 & 0.0000 \\
\hline Iceland & 43.6784 & -0.0018 & 43.6802 & 0.0000 & Uzbekistan & 59.7668 & 0.0144 & 59.7524 & 0.0000 \\
\hline Iran. & 57.3186 & -0.0441 & 57.3627 & 0.0000 & Venezuela & 39.7525 & 0.0004 & 39.6965 & -0.0556 \\
\hline Israel & 28.9126 & -0.0198 & 28.9324 & 0.0000 & Vietnam & 52.7845 & 0.0001 & 52.7732 & -0.0112 \\
\hline Italy & 35.7060 & 0.0042 & 35.6679 & -0.0339 & Zimbabwe & 27.0798 & 0.0138 & 27.1261 & 0.0601 \\
\hline
\end{tabular}


Table 8. Trade-off effects on the nitrous oxide $\left(z_{2}^{b}\right)$ when $h=-0.5$ and $d=(1,1)$.

\begin{tabular}{|c|c|c|c|c|c|c|c|c|c|}
\hline \multirow{2}{*}{$\mathrm{DMU}_{j}$} & \multicolumn{2}{|c|}{ w.r.t $y_{1}^{g}$} & \multicolumn{2}{|c|}{ w.r.t $y_{2}^{g}$} & \multirow{2}{*}{$\mathrm{DMU}_{j}$} & \multicolumn{2}{|c|}{ w.r.t $y_{1}^{g}$} & \multicolumn{2}{|c|}{ w.r.t $y_{2}^{g}$} \\
\hline & $z_{2}^{b-}$ & $\tau_{21}^{-}$ & $z_{2}^{b-}$ & $\tau_{22}^{-}$ & & $z_{2}^{b-}$ & $\tau_{21}^{-}$ & $z_{2}^{b-}$ & $\tau_{22}^{-}$ \\
\hline Albania & 36.9676 & -0.1287 & 37.0963 & 0.0000 & Korea, Rep. & 42.2033 & -0.0002 & 42.2340 & 0.0305 \\
\hline Armenia & 16.4509 & -0.0586 & 16.5095 & 0.0000 & Latvia & 34.1123 & -0.0690 & 33.9119 & -0.2694 \\
\hline Australia & 49.3314 & 0.0034 & 49.3280 & 0.0000 & Lebanon & 64.8823 & 0.0408 & 64.8415 & 0.0000 \\
\hline Austria & 38.4108 & -0.0006 & 38.4927 & 0.0812 & Luxembourg & 1.1175 & -0.0145 & 1.0476 & -0.0844 \\
\hline Azerbaijan & 11.0384 & 0.0073 & 11.0310 & 0.0000 & $\begin{array}{c}\text { North } \\
\text { Macedonia }\end{array}$ & 28.2153 & -0.0064 & 28.1942 & -0.0275 \\
\hline Bangladesh & 54.2828 & -0.0028 & 54.2856 & 0.0000 & Madagascar & 40.0712 & 0.0034 & 40.0829 & 0.0151 \\
\hline Barbados & 8.4524 & 0.0219 & 8.7039 & 0.2734 & Malawi & 3.0319 & -0.0799 & 2.7177 & -0.3941 \\
\hline Belarus & 40.7744 & -0.0154 & 40.8790 & 0.0892 & Malta & 8.2990 & -0.0085 & 8.3075 & 0.0000 \\
\hline Belize & 33.9164 & 0.0000 & 33.8727 & -0.0437 & Mauritius & 38.8434 & 0.0502 & 38.7932 & 0.0000 \\
\hline Benin & 0.0000 & 0.0000 & 0.0000 & 0.0000 & Mexico & 28.0033 & 0.0033 & 27.9731 & -0.0269 \\
\hline Bolivia & 15.4104 & -0.0005 & 15.4113 & 0.0004 & Moldova & 39.1064 & 0.0090 & 39.1879 & 0.0905 \\
\hline Bosnia and & & & & & & & & & \\
\hline $\begin{array}{l}\text { Herzegov- } \\
\text { ina }\end{array}$ & 62.5537 & -0.0151 & 62.6565 & 0.0877 & Mongolia & 0.0000 & 0.0000 & 0.0000 & 0.0000 \\
\hline Botswana & 46.6598 & -0.0158 & 46.6756 & 0.0000 & Namibia & 0.0000 & 0.0000 & 0.0000 & 0.0000 \\
\hline Bulgaria & 31.7221 & 0.0210 & 31.7010 & 0.0000 & Nepal & 43.0803 & 0.0178 & 43.0488 & -0.0137 \\
\hline Chile & 35.5376 & 0.0253 & 35.5122 & 0.0000 & Netherlands & 38.1945 & 0.0091 & 38.1854 & 0.0000 \\
\hline Costa Rica & 45.8673 & 0.0000 & 45.8726 & 0.0053 & Nicaragua & 24.3450 & -0.0259 & 24.2431 & -0.1278 \\
\hline $\begin{array}{c}\text { Cote } \\
\text { d'Ivoire }\end{array}$ & 3.4958 & 0.0262 & 3.4495 & -0.0202 & Norway & 41.9786 & -0.0009 & 42.1022 & 0.1227 \\
\hline Croatia & 26.9355 & 0.0177 & 26.9179 & 0.0000 & Oman & 26.1715 & 0.0063 & 26.1652 & 0.0000 \\
\hline Cuba & 16.9674 & -0.0095 & 16.9217 & -0.0552 & Panama & 0.0000 & 0.0000 & 0.0000 & 0.0000 \\
\hline Cyprus & 42.0092 & 0.0000 & 42.2018 & 0.1926 & Paraguay & 31.7452 & -0.0656 & 31.8491 & 0.0382 \\
\hline $\begin{array}{c}\text { Czech } \\
\text { Republic }\end{array}$ & 65.3085 & 0.0286 & 65.2799 & 0.0000 & Philippines & 56.6833 & 0.0225 & 56.6609 & 0.0000 \\
\hline Denmark & 58.9398 & 0.0029 & 58.9369 & 0.0000 & Poland & 34.5494 & 0.0282 & 34.5212 & 0.0000 \\
\hline $\begin{array}{l}\text { Dominican } \\
\text { Republic }\end{array}$ & 18.3667 & -0.0405 & 18.4072 & 0.0000 & Portugal & 38.1966 & 0.0296 & 38.1670 & 0.0000 \\
\hline Ecuador & 54.8172 & -0.0003 & 54.8602 & 0.0427 & Romania & 5.6652 & -0.0155 & 5.6028 & -0.0780 \\
\hline Egypt & 41.7122 & 0.0198 & 41.6924 & 0.0000 & Rwanda & 11.4028 & -0.0262 & 11.3085 & -0.1206 \\
\hline El Salvador & 30.9846 & 0.0044 & 30.9802 & 0.0000 & $\begin{array}{l}\text { Saudi } \\
\text { Arabia }\end{array}$ & 18.4018 & 0.0296 & 29.9140 & 11.5419 \\
\hline Ethiopia & 24.9627 & 10.4356 & 14.5271 & 0.0000 & Senegal & 0.0000 & 0.0000 & 0.0000 & 0.0000 \\
\hline Fiji & 21.6796 & -0.0134 & 21.6349 & -0.0581 & Slovak Rep. & 1.8180 & -0.0198 & 1.9530 & 0.1151 \\
\hline France & 39.4887 & -0.0007 & 39.5241 & 0.0347 & Slovenia & 35.5002 & 0.0000 & 35.4371 & -0.0631 \\
\hline $\begin{array}{l}\text { Gambia, } \\
\text { The }\end{array}$ & 0.3101 & -0.0645 & 0.0887 & -0.2859 & Spain & 5.7754 & 0.0139 & 5.6474 & -0.1141 \\
\hline Georgia & 25.5848 & 0.0000 & 25.6461 & 0.0613 & Sri Lanka & 33.7539 & 0.0116 & 33.7423 & 0.0000 \\
\hline Germany & 10.6897 & 0.0208 & 10.6618 & -0.0072 & St. Lucia & 1.6713 & -0.0305 & 1.8793 & 0.1774 \\
\hline Ghana & 4.6209 & -0.0911 & 4.2629 & -0.4492 & Switzerland & 41.0528 & -0.0009 & 41.1728 & 0.1191 \\
\hline Greece & 41.4223 & -0.0398 & 41.6934 & 0.2313 & Thailand & 38.9607 & 0.0268 & 38.9149 & -0.0190 \\
\hline Guatemala & 24.9869 & -0.0154 & 25.0023 & 0.0000 & Tunisia & 8.8217 & -0.0389 & 8.8606 & 0.0000 \\
\hline Guinea & 0.0000 & 0.0000 & 0.0000 & 0.0000 & Turkey & 48.7023 & 0.0235 & 48.6787 & 0.0000 \\
\hline Honduras & 41.7889 & 0.0094 & 41.7795 & 0.0000 & Uganda & 0.0000 & 0.0000 & 0.0000 & 0.0000 \\
\hline Hungary & 88.6828 & 0.0311 & 88.6517 & 0.0000 & UAE & 27.1187 & 0.0376 & 27.2035 & 0.1223 \\
\hline Iceland & 43.6820 & 0.0018 & 43.6802 & 0.0000 & UK & 23.7867 & 0.0134 & 23.7733 & 0.0000 \\
\hline Iran & 57.4069 & 0.0443 & 19.6190 & -37.7437 & Uzbekistan & 59.7380 & -0.0145 & 16.7369 & -43.0156 \\
\hline Israel & 28.9523 & 0.0199 & 28.9324 & 0.0000 & Venezuela & 39.7516 & -0.0004 & 39.8082 & 0.0562 \\
\hline Italy & 35.6976 & -0.0042 & 35.7364 & 0.0346 & Vietnam & 52.7843 & -0.0001 & 52.7957 & 0.0113 \\
\hline Jamaica & 0.0000 & 0.0000 & 0.0000 & 0.0000 & Zambia & 0.0000 & 0.0000 & 0.0000 & 0.0000 \\
\hline Jordan & 44.6324 & -0.0005 & 44.6329 & 0.0000 & Zimbabwe & 27.0521 & -0.0139 & 27.0044 & -0.0615 \\
\hline
\end{tabular}

Now, if we analyze the results in Table 5 when $h=+0.5$ with $d=(1,1), 67$ countries out of 136 showed the trade-off response for methane emission $\left(z_{1}^{b}\right)$. Each good-output $\left(y_{1}^{g}\right.$ or $\left.y_{2}^{g}\right)$ is increased individually (one at a time) by 0.5 unit, the excessive bad-output is 
either increased, decreased, or remained the same. Let us examine three sample DMUs for understanding sake. Table 5 shows that if we increase $y_{1}^{g}$ for Austria by 0.5 (i.e., from 89.48 to 89.98), see Table A1 in Appendix A, the methane emission $\left(z_{1}^{b+}\right)$ is reduced to 45.9628 with the trade-off $\tau_{11}^{+}=-0.0018$. However, when $y_{2}^{g}$ is increased with $h=+0.5$ from 46.99 to 47.49 , there is no change observed (i.e., $\tau_{12}^{+}=0$ ), which shows that there exists a lack of forest area. Similarly, when considering Pakistan, $z_{1}^{b+}$ is seen with the reduction to 30.4262 with $\tau_{12}^{+}=-0.0306$ (when $y_{1}^{g}$ is increased from 86.93 to 87.43 , see Table A1, Appendix A), while there is no change when the trade-off is applied to $y_{2}^{g}$. In the case of Iraq, there is no change in methane emission $z_{1}^{b+}$, when crop production $y_{1}^{g}$ is supposedly increased by $h=+0.5$, i.e., from 84.43 to 83.93 (showing the under-production), but there is an increase in $z_{1}^{b+}$ with an increase in $y_{2}^{g}$ indicating operational deficiencies i.e., massive soil erosion, etc. It is important to note that the DMUs with an increase in an undesirable-output have a minimal impact. It does not degrade the overall efficiency of those DMUs from their current efficiency point. This is due to the selection of feasible points $(h=+0.5,-0.5)$. The good thing about this trade-off is that there are few DMUs (Bolivia, Madagascar, and UAE, etc.) which improve the efficiency with both undesirable outputs $\left(y_{1}^{g}\right.$ and $\left.y_{2}^{g}\right)$. It can easily be seen in Figure 1 that the trade-off in crop production $\left(y_{1}^{g}\right)$ produces better efficiency mostly avoiding the extreme point as compared to the forest area $\left(y_{2}^{g}\right)$.

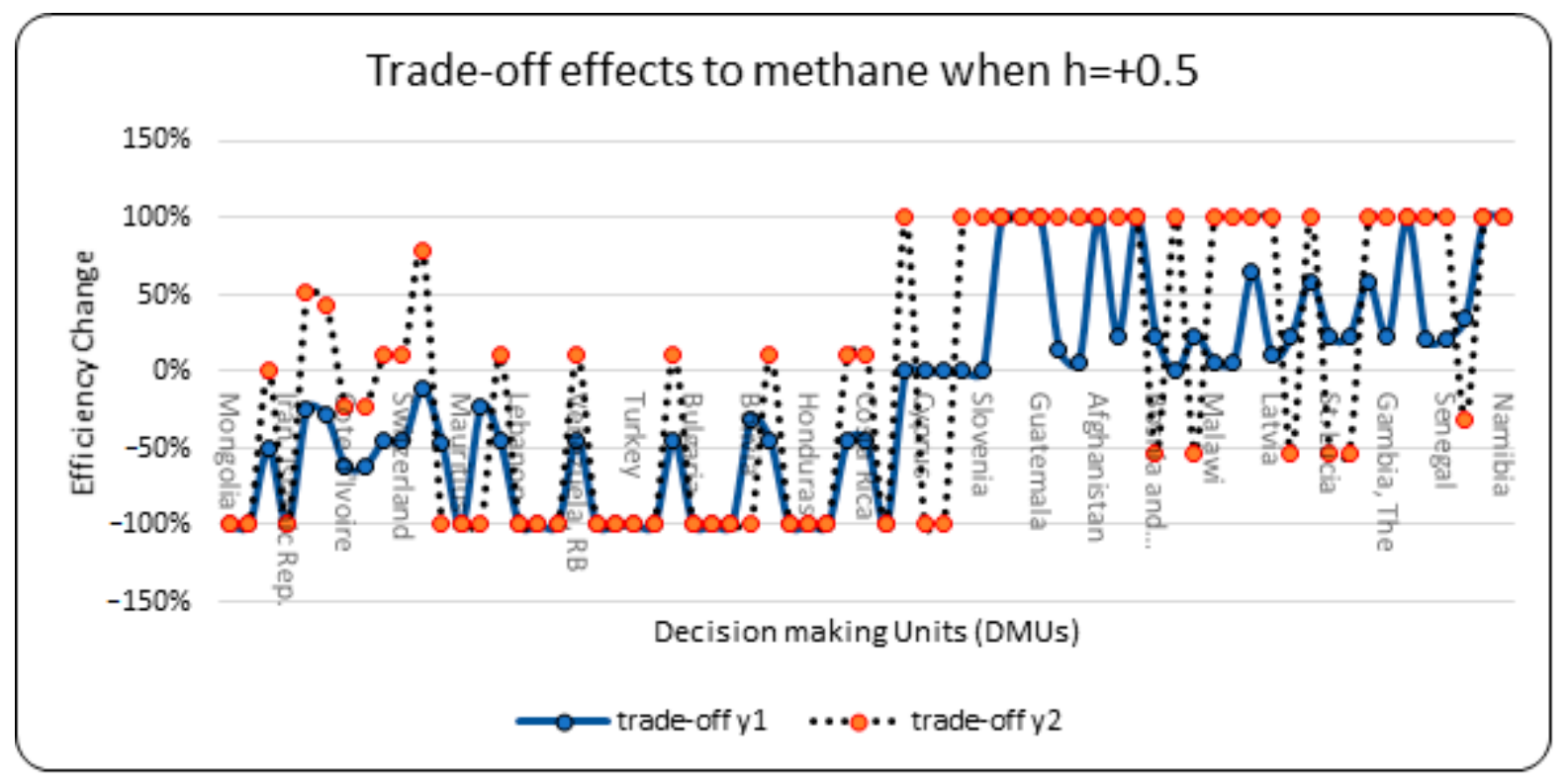

Figure 1. Trade-off effects on methane emissions $\left(z_{1}^{b}\right)$, when $h=+0.5$.

Next, we look for the effects on methane emissions $\left(z_{1}^{b}\right)$ in Table 6, when the trade-off is applied with $h=-0.5$ to $y_{1}^{g}$ and $y_{2}^{g}$. Again, if we trade-off Austria's $y_{1}^{g}$ with $h=-0.5$, i.e., from 49.48 to 48.98 , the $z_{1}^{b-}$ increases minimal with trade-off rate $\tau_{11}^{-}=0.0018$, but it decreases as $z_{1}^{b-}=45.9624$ with trade-off rate $\tau_{12}^{-}=-0.0023$ when $y_{2}^{g}$ is reduced by $h=-0.5$ (from 46.99 to 46.49). Pakistan's situation does not fit this trade-off, as it increases or does not change with $y_{1}^{g}$ and $y_{2}^{g}$, respectively. In Iraq's case, it does not make any difference with either reduction (crop production or forest area). Likewise, all the other DMUs can be checked in Table 6. With this trade-off (i.e., when $h=-0.5$ ), again we found that $y_{1}^{g}$ produces a better result as compared to $y_{2}^{g}$ (that is when the crop production is improved as compared to the improvement in the forest area), see Figure 2. It does not imply that the area of crop needs to be expanded. Of course, expending crop area will cause more agricultural erosion and maintenance, rather better production techniques for more production with the same crop area is the answer here. 


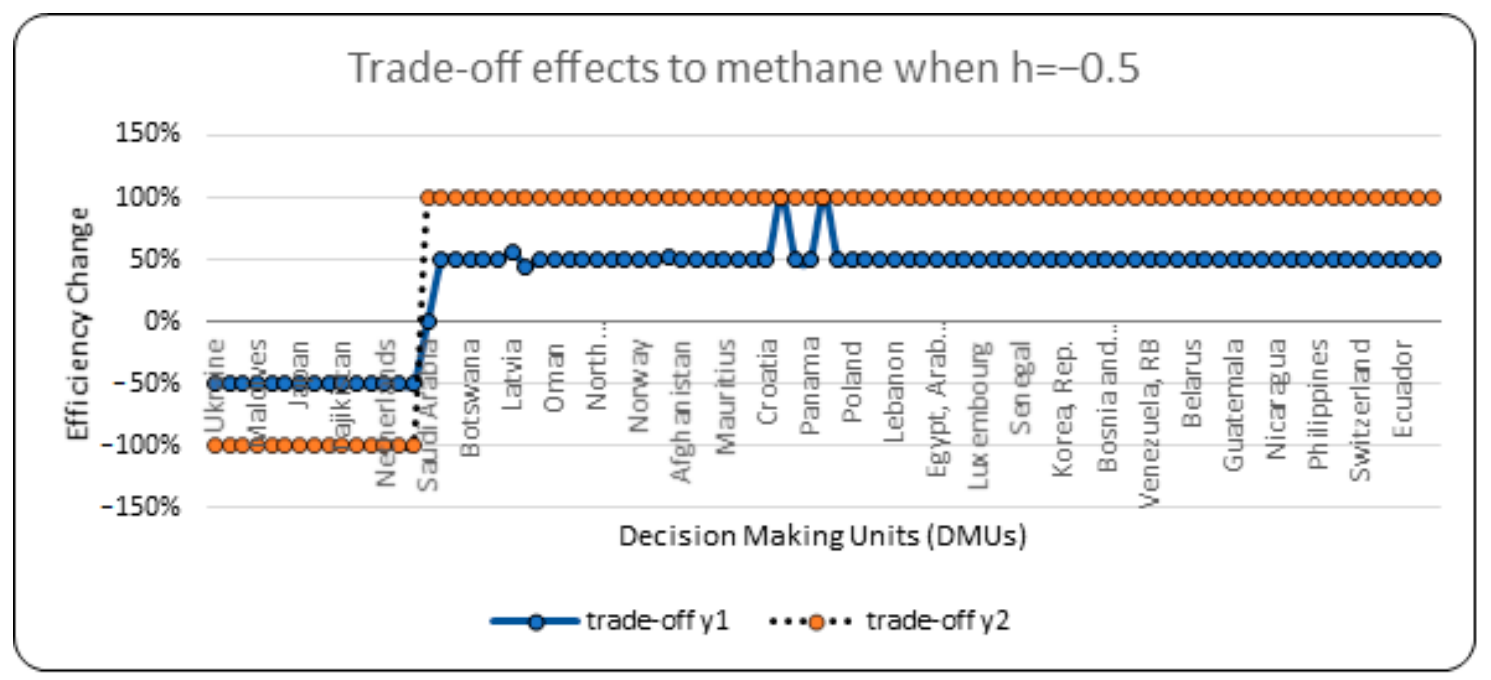

Figure 2. Trade-off effects on methane emissions $\left(z_{1}^{b}\right)$, when $h=-0.5$.

Moving on to the second undesirable-output, the nitrous oxide $z_{2}^{b}$. We again performed the same experiment, when $\mathrm{h}=+0.5$ with $\mathrm{d}=(1,1)$, and the trade-off impact can be seen in Figure 3. However, quantitatively, Table 7 shows that if we increase desirable-output $y_{1}^{g}$ for Austria by +0.5 (i.e., from 89.48 to 89.98 ), see Table A in Appendix A, the nitrous oxide emissions $\left(z_{2}^{b+}\right)$ are not facing much rise i.e., 38.4120 with trade-off $\tau_{21}^{+}=0.0006$. However, when $y_{2}^{g}$ is increased from 46.99 to 47.49 with $h=+0.5$ emissions are reduced to 38.3310 with $\tau_{22}^{+}=-0.0804$, which shows that increasing the forest area would amply reduce the nitrous oxide emissions $\left(z_{2}^{b+}\right)$. Similarly, when considering Pakistan, there is no effect at all on $z_{2}^{b+}$ with either trade-off $y_{1}^{g}$ or $y_{2}^{g}$ (this is why the DMU "Pakistan" cannot be found in Table 7). In the case of Iraq, again there is no change in nitrous oxide emission $z_{2}^{b+}$, when crop production $y_{1}^{g}$ is increased by $h=+0.5$, i.e., from 84.43 to 83.93 or when forest area $y_{2}^{g}$ is increased from 1.91 to 2.41, see Table A in Appendix A. The overall efficiency is best seen when the trade-off is applied to $y_{1}^{g}$, it has rather a stable line as compared with $y_{2}^{g}$.

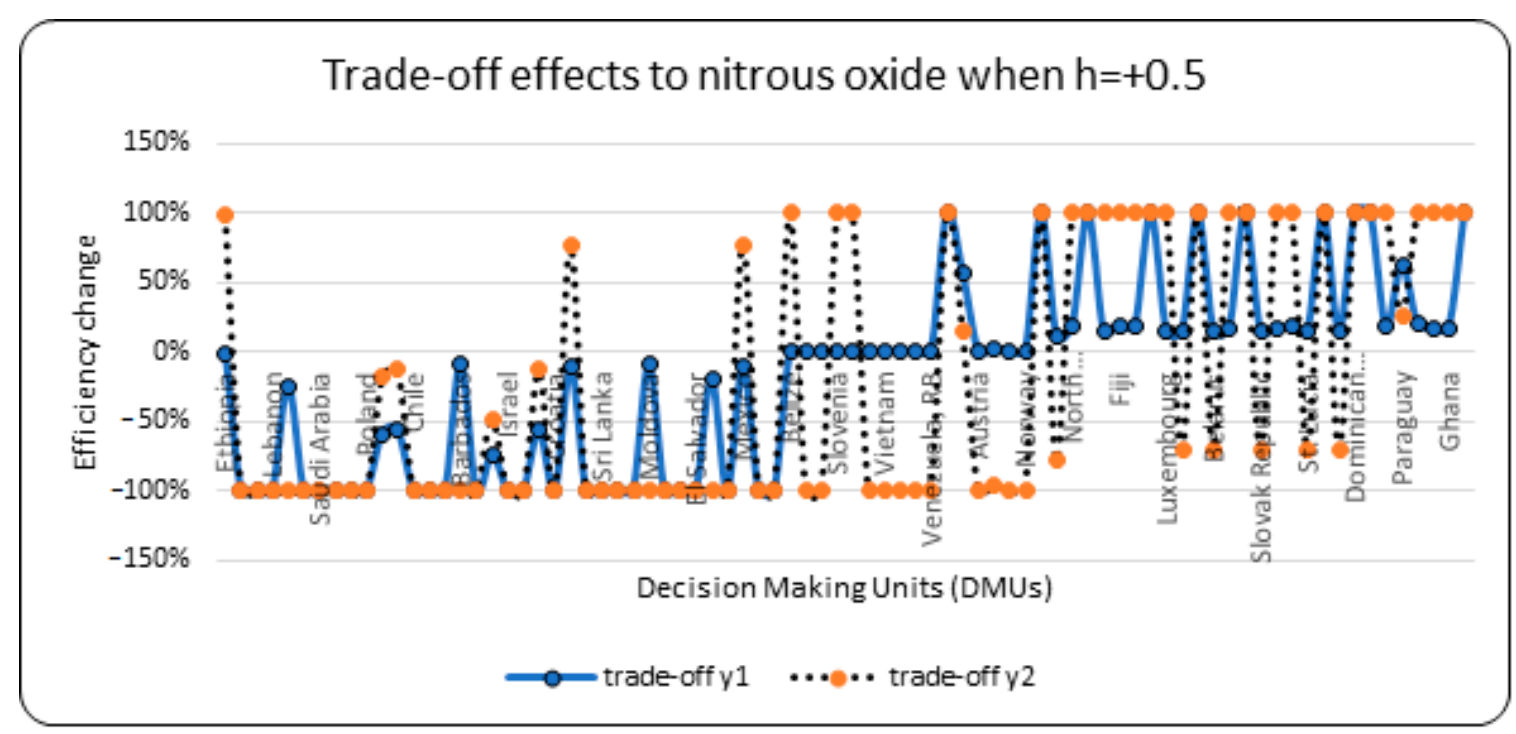

Figure 3. Trade-off effects on the nitrous oxide emissions $\left(z_{2}^{b}\right)$, when $h=+0.5$.

Next, we look for the effects on nitrous oxide emissions $\left(z_{2}^{b}\right)$ in Table 8, when the tradeoff is applied to $y_{1}^{g}$ and $y_{2}^{g}$ with $h=-0.5$. Again, if we trade-off Austria's $y_{1}^{g}$ with $h=-0.5$. i.e., from 49.48 to 48.98 , the $z_{2}^{b-}$ decreases to 38.4108 with trade-off rate $\tau_{21}^{-}=-0.0006$, but it 
increases as $z_{1}^{b-}=38.4927$ with trade-off rate $\tau_{12}^{-}=0.0812$ when $y_{2}^{g}$ is reduced by $h=-0.5$ (from 46.99 to 46.49), see Table A in Appendix A. Again, Pakistan's situation does not show any improvement with the trade-off $(h=-0.5)$ to $y_{1}^{g}$ and $y_{2}^{g}$, respectively. In Iraq's case, it again does not make any difference with either reduction (such as crop production and forest area). However, in this trade-off experiment, we found some huge declines in nitrous oxide emissions, such as Iran $\left(\tau_{22}^{-}=-37.7437\right)$ and Uzbekistan $\left(\tau_{22}^{-}=-43.0156\right)$. Likewise, all the other DMUs can be checked in Table 8. Considering the overall DMUs efficiency (which collectively make a significant contribution to the world ecology), we again find that $y_{1}^{g}$ produces a better result as compared to $y_{2}^{g}$ (see Figure 4).

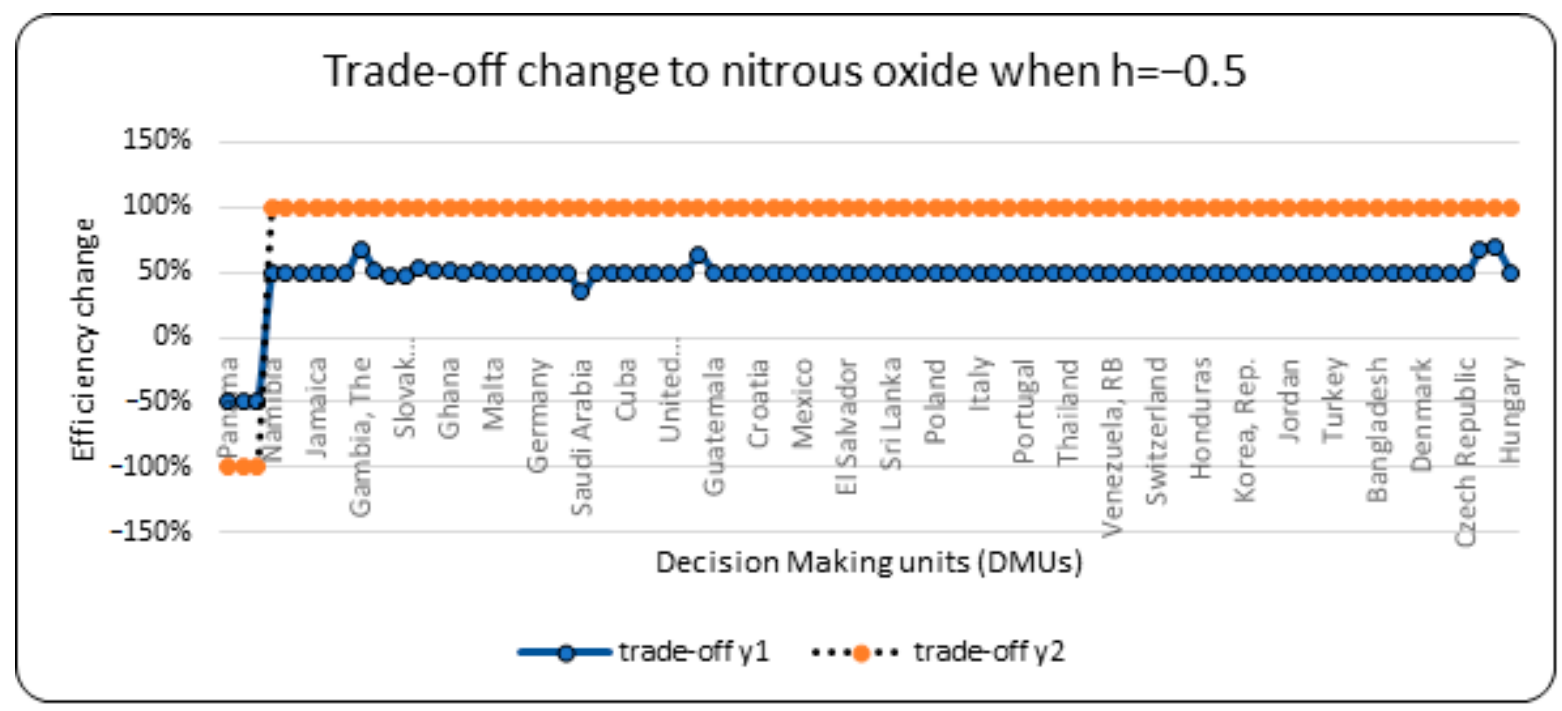

Figure 4. Trade-off effects on the nitrous oxide emissions $\left(z_{2}^{b}\right)$, when $h=-0.5$.

Since we applied the same trade-off program with same trade-off margin (i.e., $h=$ $\pm 0.5)$ to each desirable-output $\left(y_{1}^{g}\right.$ and $\left.y_{2}^{g}\right)$ to see the efficient change in each undesirableoutput $\left(z_{1}^{b}\right.$ and $\left.z_{2}^{b}\right)$, we established at each step that trade-off in $y_{1}^{g}$ produced a better result in all four experiments above. Out of the four experiments, two experiments of trade-off $h=+0.5$ produced more efficient results among undesirable-outputs $\left(z_{1}^{b}\right.$ and $\left.z_{2}^{b}\right)$, see Figures 1 and 3. Now, if we compare it with the original emission values of methane $z_{1}^{b}$ and nitrous oxide $z_{2}^{b}$, we can authenticate that trade-off point at $h=+0.5$ is the optimal trade-off point to achieve indirect efficiency, see Figure 5. There is an improvement in almost all the inefficient countries except those with zero change (Algeria, Angola, and Argentina, etc.). There are no deficiencies to the unchanged DMUs, either they were already at the efficient frontier or below the frontier line. Figure 5 shows the change in emissions in both the methane and the nitrous oxide with trade-off $h=0$ to $h=+0.5$. Resultantly, we found that trade-off in the better crop production quantity makes the maximum result out of it. The marginal trade-off is found to be the best and fastest policy action for the majority of the ecological deficiency solutions. 


\section{Trade-off effects on methane and nitrousoxide emissions}

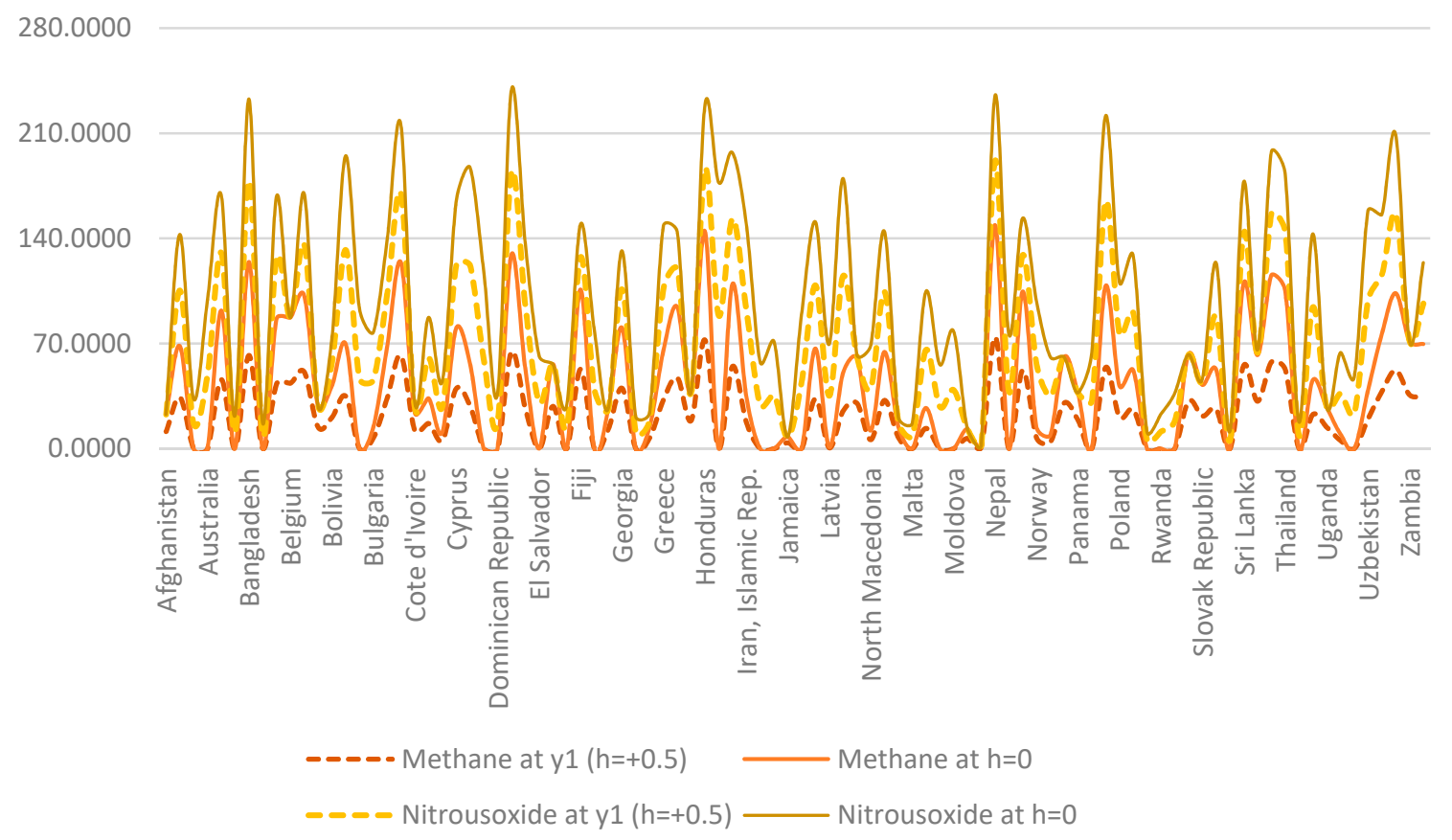

Figure 5. The efficiency change with trade-off $h=+0.5$ values with desirable outputs $\left(y_{1}^{g+}\right)$.

\section{Conclusions}

Agriculture is becoming the center of concern in terms of food security and ecological betterment. This paper produces unique trade-off methodology unlike the existing tradeoffs which uses theoretical logic and hypotheses to hypothesize the maximum change, i.e., when we change one variable, other performance variables will have an impact. However, we used the practical example to the marginal trade-off in the agricultural ecological context.

The DEA weighted slack based measurement was used to analyze the trade-off behavior between undesirable-output variables in terms of efficiencies. We obtained different results from several experiments and found the best efficiency point out of each experiment. It was found that it is possible to get different trade-off margins for each experiment. In our case, 136 developing countries were evaluated for their agro-ecological efficiency, where 31 countries were found efficient. The inefficient countries were further examined with the trade-off model by selecting the marginal range of $h= \pm 0.5$. The interesting point is that all the values between this margin produced almost the same result. The obtained results prove that the trade-off of $h=+0.5$, in good-output (i.e., crop production $y_{1}^{g}$ ) produces efficient results, which means that it not only increases the good-output (which is desirable), but also reduces the undesirable emissions (i.e., methane emission $z_{1}^{b}$ and nitrous oxide emissions $z_{2}^{b}$ ). This implies that the improved crop production (without increasing the agricultural land area) with effective managerial technique can help in reaching the efficiency frontier boundary. Therefore, we suggest that adopting the crop efficient production technique can yield the best ecological results and also improve the agricultural economic viability.

The results obtained using real numerical data prove this method's applicability in different agricultural energy efficiency assessments and improvements. This can be very helpful for the policy designers and the decision makers, who are responsible for the resource consumption and production at both the micro and macro level operations. 
However, this study is made limited to output variables (i.e., two good and two bad variables), which is different from the previous studies. The number of variables adopted in this study is subject to the data availability, therefore indicates the study limitations. Future research can be expanded by considering more output variables or more comprehensive data (including input variables, for example), such as applying marginal trade-offs to more input variables and examining the behavior of alternate output variables to find better results. Moreover, the application research in the conditional DEA method needs to be explored further.

Author Contributions: Conceptualization, A.R. and T.G.J.; methodology, U.A. and M.A.; software, U.A.; formal analysis, U.A.; data curation, A.R.; writing-original draft preparation, A.R., T.G.J. and U.A.; writing-review anediting, J.O. and J.P.; supervision, M.A. and J.O.; project administration, U.A. and M.A.; funding acquisition, J.O. and J.P. All authors have read and agreed to the published version of the manuscript."

Funding: Project no. 132805 was implemented with support provided from the National Research, Development, and Innovation Fund of Hungary, financed under the K_19 funding scheme and supported by the János Bolyai Research Scholarship of the Hungarian Academy of Sciences.

Data Availability Statement: All the variables and related data is collected from the World Bank ( https:/ / data.worldbank.org/). The countries with missing data was eliminated for better results and finally 136 countries were included in the analysis. The Section 4.1 further details the variable collected.

Conflicts of Interest: The authors declare no conflict of interest. The funders had no role in the design of the study; in the collection, analyses, or interpretation of data; in the writing of the manuscript, or in the decision to publish the results.

\section{Appendix A}

The dataset of 136 developing countries. The dataset shows the four inputs namely freshwater consumption $\left(x_{1}\right)$, agricultural land area $\left(x_{2}\right)$, fertilizer consumption $\left(x_{3}\right)$, and total agricultural labor $\left(x_{4}\right)$. Additionally, the four outputs are composed of two desirableoutputs namely production $\left(y_{1}^{g}\right)$ and forest area $\left(y_{2}^{g}\right)$, whereas, the two undesirable-outputs are methane emissions $\left(z_{1}^{b}\right)$ and nitrous oxide emissions $\left(z_{2}^{b}\right)$.

Table A1. Developing countries.

\begin{tabular}{lcccccccc}
\hline \multicolumn{1}{c}{ Country } & $x_{1}$ & $x_{2}$ & $x_{3}$ & $x_{4}$ & $y_{1}^{g}$ & $y_{2}^{g}$ & $z_{1}^{b}$ & $z_{2}^{b}$ \\
\hline Afghanistan & 47.20 & 58.13 & 12.20 & 42.84 & 160.98 & 2.07 & 65.51 & 68.93 \\
Albania & 26.90 & 44.56 & 126.10 & 36.69 & 211.23 & 28.15 & 83.01 & 97.37 \\
Algeria & 11.30 & 17.64 & 22.30 & 9.86 & 202.71 & 0.92 & 8.05 & 49.48 \\
Angola & 148.00 & 48.02 & 8.00 & 50.38 & 283.95 & 46.02 & 15.05 & 78.09 \\
Antigua and Barbuda & 0.10 & 20.45 & 13.90 & 0.01 & 110.76 & 22.16 & 65.79 & 48.81 \\
Argentina & 292.00 & 57.89 & 50.30 & 0.09 & 149.88 & 9.49 & 66.01 & 99.85 \\
Armenia & 6.90 & 64.60 & 110.50 & 29.64 & 173.67 & 11.64 & 21.57 & 92.19 \\
Australia & 492.00 & 47.35 & 68.10 & 2.56 & 115.68 & 15.91 & 52.26 & 93.26 \\
Austria & 55.00 & 31.95 & 141.80 & 3.58 & 89.48 & 46.99 & 46.41 & 66.86 \\
Azerbaijan & 8.10 & 57.81 & 14.10 & 35.87 & 129.60 & 14.92 & 35.07 & 81.19 \\
Bahamas, The & 0.70 & 1.47 & 144.00 & 2.14 & 162.44 & 51.45 & 1.39 & 30.85 \\
Bangladesh & 105.00 & 69.56 & 289.40 & 38.58 & 167.26 & 10.90 & 63.56 & 86.05 \\
Barbados & 0.10 & 23.67 & 113.80 & 2.63 & 47.83 & 14.65 & 18.32 & 32.92 \\
Belarus & 34.00 & 41.95 & 146.60 & 11.02 & 114.46 & 43.01 & 48.38 & 71.53 \\
Belgium & 12.00 & 43.38 & 318.50 & 0.96 & 94.78 & 22.75 & 74.20 & 30.75 \\
Belize & 15.30 & 7.16 & 466.20 & 16.85 & 100.19 & 58.88 & 52.59 & 69.22 \\
Benin & 10.30 & 33.91 & 14.70 & 38.58 & 169.68 & 36.65 & 49.25 & 74.39 \\
Bhutan & 78.00 & 13.49 & 13.30 & 55.31 & 118.40 & 74.53 & 30.03 & 38.69 \\
\hline
\end{tabular}


Table A1. Cont.

\begin{tabular}{|c|c|c|c|c|c|c|c|c|}
\hline Country & $x_{1}$ & $x_{2}$ & $x_{3}$ & $x_{4}$ & $y_{1}^{g}$ & $y_{2}^{g}$ & $z_{1}^{b}$ & $z_{2}^{b}$ \\
\hline Bolivia & 303.50 & 35.12 & 7.60 & 30.71 & 166.59 & 49.10 & 39.90 & 83.99 \\
\hline $\begin{array}{l}\text { Bosnia and } \\
\text { Herzegovina }\end{array}$ & 35.50 & 42.55 & 131.80 & 15.38 & 107.60 & 42.68 & 41.18 & 94.47 \\
\hline Botswana & 2.40 & 45.76 & 89.60 & 20.69 & 130.65 & 18.38 & 75.41 & \#\#\#\#\# \\
\hline Brunei Darussalam & 8.50 & 3.04 & 141.80 & 1.36 & 97.74 & 70.90 & 0.14 & 43.09 \\
\hline Bulgaria & 21.00 & 44.94 & 125.50 & 6.39 & 126.55 & 36.43 & 8.08 & 55.74 \\
\hline Burkina Faso & 12.50 & 46.98 & 21.80 & 25.23 & 159.82 & 18.77 & 78.81 & 85.75 \\
\hline Burundi & 10.10 & 80.18 & 5.40 & 92.02 & 105.27 & 12.55 & 14.61 & 54.39 \\
\hline Cambodia & 120.60 & 32.03 & 17.40 & 32.30 & 286.48 & 50.86 & 69.18 & 25.64 \\
\hline Cameroon & 273.00 & 21.20 & 9.70 & 43.44 & 219.29 & 38.15 & 59.82 & 87.96 \\
\hline $\begin{array}{l}\text { Central African } \\
\text { Republic }\end{array}$ & 141.00 & 8.09 & 0.30 & 77.32 & 123.05 & 35.49 & 74.04 & 91.89 \\
\hline Chile & 885.00 & 21.35 & 293.80 & 9.00 & 119.23 & 24.18 & 34.72 & 58.16 \\
\hline Congo, Dem. Rep. & 900.00 & 11.66 & 2.50 & 65.43 & 113.18 & 66.76 & 21.81 & 45.92 \\
\hline Congo, Rep. & 222.00 & 31.19 & 1.80 & 34.13 & 138.20 & 65.24 & 19.43 & 74.21 \\
\hline Costa Rica & 113.00 & 34.68 & 604.90 & 12.11 & 141.52 & 56.01 & 63.01 & 79.62 \\
\hline Cote d'Ivoire & 76.80 & 66.28 & 51.70 & 40.05 & 135.19 & 32.76 & 16.58 & 30.39 \\
\hline Croatia & 37.70 & 29.23 & 119.30 & 5.96 & 132.29 & 34.57 & 17.88 & 52.04 \\
\hline Cuba & 38.10 & 59.33 & 49.40 & 17.51 & 79.02 & 33.04 & 58.80 & 72.12 \\
\hline Cyprus & 0.80 & 10.87 & 196.70 & 2.07 & 52.64 & 18.72 & 40.53 & 53.24 \\
\hline Czech Republic & 13.20 & 44.99 & 196.10 & 2.72 & 113.72 & 34.65 & 29.38 & 86.88 \\
\hline Denmark & 6.00 & 62.07 & 131.10 & 2.19 & 111.04 & 14.85 & 59.01 & \#\#\#\#\# \\
\hline Dominica & 0.20 & 35.41 & 88.10 & 0.01 & 117.44 & 56.44 & 58.32 & 47.09 \\
\hline Dominican Republic & 23.50 & 47.78 & 88.10 & 9.02 & 177.43 & 44.39 & 53.24 & 74.37 \\
\hline Ecuador & 442.40 & 23.86 & 345.40 & 29.20 & 123.31 & 49.33 & 65.49 & 84.55 \\
\hline Egypt, Arab Rep. & 1.80 & 3.90 & 649.20 & 23.79 & 128.62 & 0.08 & 27.94 & 66.12 \\
\hline El Salvador & 15.60 & 76.08 & 132.40 & 16.28 & 115.13 & 12.06 & 50.74 & 74.16 \\
\hline Eritrea & 2.80 & 75.47 & 2.80 & 61.21 & 81.25 & 14.78 & 66.98 & 83.04 \\
\hline Estonia & 12.70 & 24.85 & 112.70 & 3.18 & 197.25 & 51.16 & 19.65 & 70.36 \\
\hline Ethiopia & 122.00 & 38.85 & 14.40 & 66.13 & 232.09 & 12.00 & 65.84 & 87.96 \\
\hline Fiji & 28.60 & 23.19 & 46.00 & 36.26 & 67.64 & 55.84 & 79.11 & 89.39 \\
\hline Finland & 107.00 & 7.55 & 80.50 & 3.61 & 95.19 & 73.09 & 15.27 & 35.38 \\
\hline France & 200.00 & 51.87 & 163.10 & 2.44 & 96.06 & 31.92 & 41.70 & 81.26 \\
\hline Gabon & 164.00 & 20.03 & 26.80 & 32.83 & 135.50 & 89.45 & 3.17 & 16.28 \\
\hline Gambia, The & 3.00 & 65.04 & 1.20 & 27.12 & 107.13 & 48.98 & 53.49 & 85.37 \\
\hline Georgia & 58.10 & 32.25 & 170.80 & 41.82 & 69.55 & 41.03 & 41.36 & 50.19 \\
\hline Germany & 107.00 & 47.45 & 197.20 & 1.21 & 103.59 & 32.79 & 54.16 & 55.19 \\
\hline Ghana & 30.30 & 71.16 & 20.90 & 29.26 & 175.83 & 41.55 & 36.14 & 95.20 \\
\hline Greece & 58.00 & 46.46 & 123.00 & 11.98 & 85.03 & 32.47 & 36.80 & 65.00 \\
\hline Guatemala & 109.20 & 32.28 & 303.20 & 31.49 & 188.64 & 31.51 & 48.84 & 60.83 \\
\hline Guinea & 226.00 & 60.19 & 1.60 & 61.74 & 147.09 & 25.35 & 47.48 & 61.37 \\
\hline Guyana & 241.00 & 8.51 & 44.60 & 17.14 & 131.40 & 83.85 & 72.14 & 87.35 \\
\hline Honduras & 90.70 & 29.65 & 164.30 & 30.26 & 146.42 & 37.68 & 73.66 & 69.59 \\
\hline Hungary & 6.00 & 55.52 & 128.30 & 4.70 & 89.28 & 23.30 & 24.76 & \#\#\#\#\# \\
\hline Iceland & 170.00 & 18.61 & 181.50 & 3.94 & 96.94 & 0.57 & 54.97 & 86.42 \\
\hline Iran, Islamic Rep. & 128.50 & 24.23 & 76.30 & 17.95 & 111.13 & 6.78 & 19.29 & 80.02 \\
\hline Iraq & 35.20 & 20.76 & 35.80 & 18.10 & 84.43 & 1.91 & 14.37 & 33.34 \\
\hline Israel & 0.80 & 23.93 & 280.70 & 0.92 & 103.31 & 7.65 & 32.11 & 62.06 \\
\hline Italy & 182.50 & 42.56 & 129.80 & 3.68 & 88.87 & 32.50 & 35.33 & 78.07 \\
\hline Jamaica & 10.80 & 39.63 & 57.20 & 15.93 & 102.44 & 30.81 & 48.02 & 44.59 \\
\hline Japan & 430.00 & 12.07 & 242.20 & 3.42 & 85.30 & 68.54 & 74.62 & 30.71 \\
\hline Jordan & 0.70 & 11.88 & 112.00 & 3.08 & 151.59 & 1.09 & 16.85 & 80.20 \\
\hline Kazakhstan & 64.40 & 80.89 & 4.30 & 15.80 & 166.32 & 1.22 & 18.35 & \#\#\#\#\# \\
\hline Kenya & 20.70 & 49.17 & 38.20 & 54.44 & 151.83 & 8.19 & 51.52 & 88.58 \\
\hline Korea, Rep. & 64.90 & 17.08 & 380.30 & 4.88 & 90.96 & 63.04 & 33.50 & 80.50 \\
\hline
\end{tabular}


Table A1. Cont.

\begin{tabular}{|c|c|c|c|c|c|c|c|c|}
\hline Country & $x_{1}$ & $x_{2}$ & $x_{3}$ & $x_{4}$ & $y_{1}^{g}$ & $y_{2}^{g}$ & $z_{1}^{b}$ & $z_{2}^{b}$ \\
\hline Kuwait & 0.00 & 8.52 & 750.70 & 2.01 & 245.80 & 0.38 & 1.54 & 23.55 \\
\hline Kyrgyz Republic & 48.90 & 54.54 & 31.40 & 21.17 & 114.91 & 2.92 & 68.18 & 86.83 \\
\hline Latvia & 16.90 & 32.50 & 104.20 & 6.75 & 181.48 & 54.67 & 15.05 & 95.64 \\
\hline Lebanon & 4.80 & 65.88 & 330.90 & 13.61 & 92.86 & 13.58 & 25.91 & 82.48 \\
\hline Lithuania & 15.50 & 47.32 & 131.90 & 6.87 & 204.60 & 35.63 & 23.12 & 34.02 \\
\hline Luxembourg & 1.00 & 54.56 & 262.10 & 1.01 & 77.08 & 35.68 & 83.51 & 58.98 \\
\hline North Macedonia & 5.40 & 47.30 & 79.30 & 15.38 & 131.41 & 40.40 & 37.23 & 70.64 \\
\hline Madagascar & 337.00 & 71.70 & 5.20 & 64.22 & 136.70 & 21.11 & 63.35 & \#\#\#\#\# \\
\hline Malawi & 16.10 & 65.97 & 21.60 & 43.65 & 199.30 & 32.13 & 32.19 & 88.20 \\
\hline Maldives & 0.00 & 22.12 & 314.90 & 8.47 & 54.67 & 3.33 & 0.60 & 24.95 \\
\hline Mali & 60.00 & 34.41 & 44.20 & 62.59 & 197.21 & 3.64 & 78.13 & 77.10 \\
\hline Malta & 0.10 & 32.43 & 264.60 & 0.99 & 102.31 & 1.09 & 16.76 & 54.44 \\
\hline Mauritius & 2.80 & 39.98 & 235.30 & 6.07 & 76.87 & 18.65 & 14.18 & 53.39 \\
\hline Mexico & 409.00 & 54.88 & 114.00 & 12.61 & 131.19 & 33.72 & 46.86 & 73.72 \\
\hline Moldova & 1.60 & 73.65 & 24.40 & 35.93 & 99.97 & 13.23 & 16.11 & 72.61 \\
\hline Mongolia & 34.80 & 70.00 & 40.00 & 27.42 & 362.87 & 8.61 & 94.48 & \#\#\#\#\# \\
\hline Morocco & 29.00 & 68.31 & 71.10 & 34.69 & 143.03 & 13.18 & 46.85 & 76.83 \\
\hline Mozambique & 100.30 & 64.35 & 3.70 & 70.33 & 185.40 & 47.19 & 27.30 & 26.38 \\
\hline Namibia & 6.20 & 47.13 & 26.10 & 22.13 & 128.33 & 8.08 & 90.25 & 97.74 \\
\hline Nepal & 198.20 & 28.37 & 74.10 & 65.00 & 155.00 & 24.94 & 79.99 & 76.31 \\
\hline Netherlands & 11.00 & 53.16 & 288.90 & 2.04 & 112.46 & 11.33 & 43.86 & 77.92 \\
\hline Nicaragua & 156.20 & 41.27 & 61.50 & 30.65 & 143.10 & 23.93 & 70.58 & 86.48 \\
\hline Niger & 3.50 & 38.80 & 0.40 & 75.06 & 266.68 & 0.87 & 40.66 & 71.34 \\
\hline Nigeria & 221.00 & 77.52 & 5.50 & 35.10 & 122.32 & 6.57 & 33.25 & 90.05 \\
\hline Norway & 382.00 & 2.64 & 203.90 & 2.06 & 90.60 & 33.17 & 7.87 & 61.94 \\
\hline Oman & 1.40 & 4.80 & 468.10 & 4.56 & 151.71 & 0.01 & 5.71 & 54.98 \\
\hline Pakistan & 55.00 & 46.63 & 144.30 & 36.66 & 125.41 & 1.74 & 57.11 & 78.92 \\
\hline Panama & 136.60 & 30.51 & 49.10 & 13.95 & 86.93 & 61.24 & 74.97 & 76.40 \\
\hline Papua New Guinea & 801.00 & 2.87 & 112.10 & 58.32 & 133.13 & 74.08 & 12.09 & 27.33 \\
\hline Paraguay & 117.00 & 57.43 & 110.30 & 20.13 & 198.41 & 36.32 & 82.74 & \#\#\#\#\# \\
\hline Philippines & 479.00 & 43.67 & 157.40 & 23.41 & 124.13 & 26.78 & 55.15 & 80.24 \\
\hline Poland & 53.60 & 44.05 & 172.80 & 9.23 & 114.37 & 31.17 & 21.98 & 56.24 \\
\hline Portugal & 38.00 & 38.98 & 199.40 & 5.85 & 112.00 & 34.26 & 27.09 & 59.44 \\
\hline Romania & 42.40 & 58.10 & 59.90 & 21.71 & 97.25 & 30.21 & 30.76 & 63.40 \\
\hline Rwanda & 9.50 & 73.98 & 10.90 & 62.41 & 185.02 & 21.63 & 32.50 & 83.92 \\
\hline Saudi Arabia & 2.40 & 80.64 & 176.90 & 2.40 & 72.52 & 0.45 & 5.98 & 43.69 \\
\hline Senegal & 25.80 & 47.26 & 16.40 & 30.05 & 176.82 & 42.15 & 63.84 & 87.55 \\
\hline Slovak Republic & 12.60 & 37.07 & 125.80 & 2.18 & 99.43 & 40.48 & 28.14 & 32.72 \\
\hline Slovenia & 18.70 & 34.12 & 258.90 & 5.23 & 83.12 & 62.18 & 28.05 & 72.78 \\
\hline South Africa & 44.80 & 79.36 & 58.50 & 5.09 & 118.09 & 7.62 & 24.91 & 71.23 \\
\hline Spain & 111.20 & 51.00 & 144.00 & 4.09 & 106.51 & 38.04 & 58.83 & 65.86 \\
\hline Sri Lanka & 52.80 & 46.50 & 131.90 & 24.52 & 142.66 & 32.59 & 56.81 & 60.84 \\
\hline St. Kitts and Nevis & 0.00 & 19.78 & 0.50 & 0.00 & 14.94 & 42.31 & 23.39 & 52.95 \\
\hline St. Lucia & 0.30 & 15.97 & 170.90 & 17.27 & 38.59 & 32.90 & 44.75 & 36.60 \\
\hline Suriname & 99.00 & 0.55 & 217.70 & 7.52 & 185.11 & 98.18 & 67.90 & \#\#\#\#\# \\
\hline Sweden & 171.00 & 7.33 & 96.30 & 1.65 & 116.40 & 68.87 & 24.66 & 61.85 \\
\hline Switzerland & 40.40 & 38.13 & 214.40 & 2.95 & 97.97 & 32.15 & 58.53 & 60.35 \\
\hline Tajikistan & 63.50 & 34.67 & 81.40 & 44.92 & 181.47 & 2.96 & 60.35 & 92.60 \\
\hline Tanzania & 84.00 & 47.43 & 12.60 & 65.31 & 221.61 & 50.35 & 57.99 & 88.34 \\
\hline Thailand & 224.50 & 45.17 & 161.70 & 31.61 & 133.65 & 31.84 & 54.85 & 66.14 \\
\hline Togo & 11.50 & 73.03 & 11.00 & 37.70 & 152.45 & 2.76 & 28.09 & 66.29 \\
\hline Trinidad and Tobago & 3.80 & 10.07 & 356.00 & 2.97 & 46.37 & 45.25 & 0.26 & 86.63 \\
\hline Tunisia & 4.20 & 65.45 & 59.30 & 13.03 & 135.06 & 7.14 & 22.24 & 79.08 \\
\hline Turkey & 227.00 & 48.36 & 137.70 & 18.38 & 122.29 & 15.82 & 23.85 & 71.90 \\
\hline Uganda & 39.00 & 75.72 & 1.90 & 72.67 & 75.50 & 8.88 & 56.66 & 91.70 \\
\hline Ukraine & 55.10 & 71.60 & 52.70 & 14.48 & 200.85 & 16.68 & 13.03 & 33.77 \\
\hline United Arab Emirates & 0.20 & 4.52 & 714.90 & 1.41 & 45.19 & 4.59 & 5.67 & 32.76 \\
\hline United Kingdom & 145.00 & 71.81 & 252.90 & 1.03 & 103.43 & 13.17 & 46.45 & 63.12 \\
\hline
\end{tabular}


Table A1. Cont.

\begin{tabular}{lcccccccc}
\hline \multicolumn{1}{c}{ Country } & $x_{1}$ & $x_{2}$ & $x_{3}$ & $x_{4}$ & $y_{1}^{g}$ & $y_{2}^{g}$ & $z_{1}^{b}$ & $z_{2}^{b}$ \\
\hline Uruguay & 92.20 & 81.24 & 143.70 & 8.12 & 232.20 & 11.58 & 92.76 & 94.71 \\
Uzbekistan & 16.30 & 62.57 & 232.70 & 23.92 & 187.10 & 7.59 & 21.57 & 95.29 \\
Venezuela, RB & 805.00 & 24.50 & 183.50 & 8.31 & 98.62 & 52.39 & 38.68 & 71.47 \\
Vietnam & 359.40 & 40.77 & 429.80 & 37.36 & 151.68 & 51.00 & 53.50 & 83.41 \\
Yemen, Rep. & 2.10 & 44.53 & 16.40 & 28.98 & 142.45 & 1.04 & 35.29 & 64.11 \\
Zambia & 80.20 & 32.70 & 89.60 & 48.84 & 237.04 & 64.56 & 48.98 & 63.47 \\
Zimbabwe & 12.30 & 42.78 & 22.90 & 66.54 & 96.18 & 33.64 & 66.63 & 91.40 \\
\hline
\end{tabular}

\section{Appendix B}

Table A2. Trade-off effects on the methane $\left(z_{1}^{b}\right)$ and nitrous oxide $\left(z_{2}^{b}\right)$ when $h=+0.5$ and $d=(1,1)$.

\begin{tabular}{|c|c|c|c|c|c|c|c|c|}
\hline \multirow{3}{*}{$\mathbf{D M U}_{j}$} & \multicolumn{4}{|c|}{ Methane Emissions $z_{1}^{b}$} & \multicolumn{4}{|c|}{ Nitrous Oxide Emissions $z_{2}^{b}$} \\
\hline & \multicolumn{2}{|c|}{ w.r.t $y_{1}$} & \multicolumn{2}{|c|}{$\begin{array}{c}1 \\
\text { w.r.t } y_{2}\end{array}$} & \multicolumn{2}{|c|}{ w.r.t $y_{1}$} & \multicolumn{2}{|c|}{ w.r.t $y_{2}$} \\
\hline & $z_{1}^{b+}$ & $\tau_{11}^{+}$ & $z_{1}^{b+}$ & $\tau_{12}^{+}$ & $z_{2}^{b+}$ & $\tau_{21}^{+}$ & $z_{2}^{b+}$ & $\tau_{22}^{+}$ \\
\hline Afghanistan & 11.4042 & 0.0071 & 11.3971 & 0.0000 & 0.0000 & 0.0000 & 0.0000 & 0.0000 \\
\hline Albania & 34.3414 & 0.1826 & 34.1588 & 0.0000 & 37.2237 & 0.1274 & 37.0963 & 0.0000 \\
\hline Algeria & 0.0000 & 0.0000 & 0.0000 & 0.0000 & 0.0000 & 0.0000 & 0.0000 & 0.0000 \\
\hline Angola & 0.0000 & 0.0000 & 0.0000 & 0.0000 & 0.0000 & 0.0000 & 0.0000 & 0.0000 \\
\hline Antigua/Barbuda & 0.0000 & 0.0000 & 0.0000 & 0.0000 & 0.0000 & 0.0000 & 0.0000 & 0.0000 \\
\hline Argentina & 0.0000 & 0.0000 & 0.0000 & 0.0000 & 0.0000 & 0.0000 & 0.0000 & 0.0000 \\
\hline Armenia & 0.0000 & 0.0000 & 0.0000 & 0.0000 & 16.5679 & 0.0584 & 16.5095 & 0.0000 \\
\hline Australia & 0.0000 & 0.0000 & 0.0000 & 0.0000 & 49.3246 & -0.0034 & 49.3280 & 0.0000 \\
\hline Austria & 45.9628 & -0.0018 & 45.9669 & 0.0023 & 38.4120 & 0.0006 & 38.3310 & -0.0804 \\
\hline Azerbaijan & 0.0000 & 0.0000 & 0.0000 & 0.0000 & 11.0237 & -0.0073 & 11.0310 & 0.0000 \\
\hline Bahamas & 0.0000 & 0.0000 & 0.0000 & 0.0000 & 0.0000 & 0.0000 & 0.0000 & 0.0000 \\
\hline Bangladesh & 62.1303 & 0.0001 & 62.1302 & 0.0000 & 54.2884 & 0.0028 & 54.2856 & 0.0000 \\
\hline Barbados & 0.0000 & 0.0000 & 0.0000 & 0.0000 & 8.4088 & -0.0218 & 8.1748 & -0.2558 \\
\hline Belarus & 43.2344 & 0.0124 & 43.1816 & -0.0404 & 40.8051 & 0.0154 & 40.7027 & -0.0871 \\
\hline Belgium & 43.6960 & 0.0587 & 43.6373 & 0.0000 & 0.0000 & 0.0000 & 0.0000 & 0.0000 \\
\hline Belize & 51.0052 & 0.0000 & 51.0072 & 0.0019 & 33.9164 & 0.0000 & 33.9593 & 0.0429 \\
\hline Benin & 14.3868 & 0.0667 & 14.5769 & 0.2568 & 0.0000 & 0.0000 & 0.0000 & 0.0000 \\
\hline Bhutan & 0.0000 & 0.0000 & 0.0000 & 0.0000 & 0.0000 & 0.0000 & 0.0000 & 0.0000 \\
\hline Bolivia & 20.7527 & -0.0007 & 20.7518 & -0.0015 & 15.4115 & 0.0005 & 15.4105 & -0.0004 \\
\hline Bosnia/Herzegovina & 34.9513 & 0.0122 & 34.8993 & -0.0397 & 62.5839 & 0.0151 & 62.4832 & -0.0856 \\
\hline Botswana & 0.0000 & 0.0000 & 0.0000 & 0.0000 & 46.6913 & 0.0157 & 46.6756 & 0.0000 \\
\hline Brunei Darussalam & 0.0000 & 0.0000 & 0.0000 & 0.0000 & 0.0000 & 0.0000 & 0.0000 & 0.0000 \\
\hline Bulgaria & 7.0032 & -0.0009 & 7.0042 & 0.0000 & 31.6801 & -0.0209 & 31.7010 & 0.0000 \\
\hline Burkina Faso & 0.0000 & 0.0000 & 0.0000 & 0.0000 & 0.0000 & 0.0000 & 0.0000 & 0.0000 \\
\hline Burundi & 0.0000 & 0.0000 & 0.0000 & 0.0000 & 0.0000 & 0.0000 & 0.0000 & 0.0000 \\
\hline Cambodia & 0.0000 & 0.0000 & 0.0000 & 0.0000 & 0.0000 & 0.0000 & 0.0000 & 0.0000 \\
\hline Cameroon & 0.0000 & 0.0000 & 0.0000 & 0.0000 & 0.0000 & 0.0000 & 0.0000 & 0.0000 \\
\hline Central African & 0.0000 & 0.0000 & 0.0000 & 0.0000 & 0.0000 & 0.0000 & 0.0000 & 0.0000 \\
\hline Chile & 33.6971 & -0.0011 & 33.6982 & 0.0000 & 35.4870 & -0.0252 & 35.5122 & 0.0000 \\
\hline Congo, Dem. Rep. & 0.0000 & 0.0000 & 0.0000 & 0.0000 & 0.0000 & 0.0000 & 0.0000 & 0.0000 \\
\hline Congo, Rep. & 0.0000 & 0.0000 & 0.0000 & 0.0000 & 0.0000 & 0.0000 & 0.0000 & 0.0000 \\
\hline Costa Rica & 61.9982 & -0.0001 & 61.9984 & 0.0001 & 45.8674 & 0.0000 & 45.8621 & -0.0053 \\
\hline Cote d'Ivoire & 12.0108 & -0.0042 & 12.0177 & 0.0027 & 3.4436 & -0.0260 & 3.4897 & 0.0201 \\
\hline Croatia & 16.7472 & -0.0008 & 16.7480 & 0.0000 & 26.9003 & -0.0176 & 26.9179 & 0.0000 \\
\hline Cuba & 5.5215 & 0.0240 & 5.5143 & 0.0169 & 16.9863 & 0.0094 & 17.0308 & 0.0540 \\
\hline Cyprus & 40.0264 & 0.0000 & 40.0179 & -0.0085 & 42.0092 & 0.0000 & 41.8203 & -0.1889 \\
\hline Czech Republic & 28.4054 & -0.0013 & 28.4067 & 0.0000 & 65.2515 & -0.0284 & 65.2799 & 0.0000 \\
\hline Denmark & 0.0000 & 0.0000 & 0.0000 & 0.0000 & 58.9339 & -0.0029 & 58.9369 & 0.0000 \\
\hline Dominica & 0.0000 & 0.0000 & 0.0000 & 0.0000 & 0.0000 & 0.0000 & 0.0000 & 0.0000 \\
\hline
\end{tabular}


Table A2. Cont.

\begin{tabular}{|c|c|c|c|c|c|c|c|c|}
\hline \multirow{3}{*}{$\mathbf{D M U}_{j}$} & \multicolumn{4}{|c|}{ Methane Emissions $z_{1}^{b}$} & \multicolumn{4}{|c|}{ Nitrous Oxide Emissions $z_{2}^{b}$} \\
\hline & \multicolumn{2}{|c|}{ w.r.t $y_{1}$} & \multicolumn{2}{|c|}{ w.r.t $y_{2}$} & \multicolumn{2}{|c|}{ w.r.t $y_{1}$} & \multicolumn{2}{|c|}{ w.r.t $y_{2}$} \\
\hline & $z_{1}^{b+}$ & $\tau_{11}^{+}$ & $z_{1}^{b+}$ & $\tau_{12}^{+}$ & $z_{2}^{b+}$ & $\tau_{21}^{+}$ & $z_{2}^{b+}$ & $\tau_{22}^{+}$ \\
\hline Dominican Rep. & 0.0000 & 0.0000 & 0.0000 & 0.0000 & 18.4475 & 0.0403 & 18.4072 & 0.0000 \\
\hline Ecuador & 64.6114 & -0.0010 & 64.6135 & 0.0012 & 54.8178 & 0.0003 & 54.7752 & -0.0423 \\
\hline Egypt, Arab Rep. & 26.8410 & -0.0009 & 26.8419 & 0.0000 & 41.6727 & -0.0197 & 41.6924 & 0.0000 \\
\hline El Salvador & 0.0000 & 0.0000 & 0.0000 & 0.0000 & 30.9759 & -0.0044 & 30.9802 & 0.0000 \\
\hline Eritrea & 28.1564 & 0.0090 & 28.1474 & 0.0000 & 0.0000 & 0.0000 & 0.0000 & 0.0000 \\
\hline Estonia & 0.0000 & 0.0000 & 0.0000 & 0.0000 & 0.0000 & 0.0000 & 0.0000 & 0.0000 \\
\hline Ethiopia & 0.0000 & 0.0000 & 0.0000 & 0.0000 & 14.4607 & -0.0664 & 24.8439 & 10.3167 \\
\hline Fiji & 52.9460 & -0.0077 & 52.9780 & 0.0243 & 21.7064 & 0.0134 & 21.7505 & 0.0575 \\
\hline Finland & 0.0000 & 0.0000 & 0.0000 & 0.0000 & 0.0000 & 0.0000 & 0.0000 & 0.0000 \\
\hline France & 0.0000 & 0.0000 & 0.0000 & 0.0000 & 39.4901 & 0.0007 & 39.4550 & -0.0344 \\
\hline Gabon & 0.0000 & 0.0000 & 0.0000 & 0.0000 & 0.0000 & 0.0000 & 0.0000 & 0.0000 \\
\hline Gambia, The & 12.8958 & 0.0367 & 12.9796 & 0.1206 & 0.4388 & 0.0642 & 0.6542 & 0.2795 \\
\hline Georgia & 40.2571 & 0.0000 & 40.2544 & -0.0027 & 25.5848 & 0.0000 & 25.5247 & -0.0601 \\
\hline Germany & 0.0000 & 0.0000 & 0.0000 & 0.0000 & 10.6484 & -0.0205 & 10.6761 & 0.0072 \\
\hline Ghana & 7.1747 & 0.0142 & 7.3637 & 0.2032 & 4.8027 & 0.0907 & 5.1514 & 0.4394 \\
\hline Greece & 32.6785 & 0.0322 & 32.5415 & -0.1048 & 41.5020 & 0.0399 & 41.2363 & -0.2258 \\
\hline Guatemala & 47.2316 & 0.0007 & 47.2309 & 0.0000 & 25.0176 & 0.0153 & 25.0023 & 0.0000 \\
\hline Guinea & 18.7337 & 0.0029 & 18.7488 & 0.0180 & 0.0000 & 0.0000 & 0.0000 & 0.0000 \\
\hline Guyana & 0.0000 & 0.0000 & 0.0000 & 0.0000 & 0.0000 & 0.0000 & 0.0000 & 0.0000 \\
\hline Honduras & 72.4080 & -0.0004 & 72.4084 & 0.0000 & 41.7701 & -0.0093 & 41.7795 & 0.0000 \\
\hline Hungary & 0.0000 & 0.0000 & 0.0000 & 0.0000 & 88.6208 & -0.0309 & 88.6517 & 0.0000 \\
\hline Iceland & 54.8233 & 0.0000 & 54.8234 & 0.0000 & 43.6784 & -0.0018 & 43.6802 & 0.0000 \\
\hline Iran, Islamic Rep. & 17.4403 & -0.0077 & 17.4480 & 0.0000 & 57.3186 & -0.0441 & 57.3627 & 0.0000 \\
\hline Iraq & 0.0000 & 0.0000 & 5.3387 & 5.3387 & 0.0000 & 0.0000 & 0.0000 & 0.0000 \\
\hline Israel & 0.0000 & 0.0000 & 0.0000 & 0.0000 & 28.9126 & -0.0198 & 28.9324 & 0.0000 \\
\hline Italy & 0.0000 & 0.0000 & 0.0000 & 0.0000 & 35.7060 & 0.0042 & 35.6679 & -0.0339 \\
\hline Jamaica & 3.9368 & -0.0025 & 3.9595 & 0.0202 & 0.0000 & 0.0000 & 0.0000 & 0.0000 \\
\hline Japan & 0.0000 & 0.0000 & 0.0000 & 0.0000 & 0.0000 & 0.0000 & 0.0000 & 0.0000 \\
\hline Jordan & 0.0000 & 0.0000 & 0.0000 & 0.0000 & 44.6334 & 0.0005 & 44.6329 & 0.0000 \\
\hline Kazakhstan & 0.0000 & 0.0000 & 0.0000 & 0.0000 & 0.0000 & 0.0000 & 0.0000 & 0.0000 \\
\hline Kenya & 0.0000 & 0.0000 & 0.0000 & 0.0000 & 0.0000 & 0.0000 & 0.0000 & 0.0000 \\
\hline Korea, Rep. & 33.3108 & -0.0007 & 33.3123 & 0.0008 & 42.2038 & 0.0002 & 42.1734 & -0.0302 \\
\hline Kuwait & 0.0000 & 0.0000 & 0.0000 & 0.0000 & 0.0000 & 0.0000 & 0.0000 & 0.0000 \\
\hline Kyrgyz Republic & 0.0000 & 0.0000 & 0.0000 & 0.0000 & 0.0000 & 0.0000 & 0.0000 & 0.0000 \\
\hline Latvia & 0.5898 & 0.0150 & 0.6966 & 0.1218 & 34.2498 & 0.0686 & 34.4448 & 0.2636 \\
\hline Lebanon & 25.1189 & -0.0018 & 25.1207 & 0.0000 & 64.8009 & -0.0405 & 64.8415 & 0.0000 \\
\hline Lithuania & 0.0000 & 0.0000 & 0.0000 & 0.0000 & 0.0000 & 0.0000 & 0.0000 & 0.0000 \\
\hline Luxembourg & 30.2811 & 0.0367 & 30.2703 & 0.0259 & 1.1464 & 0.0145 & 1.2145 & 0.0826 \\
\hline North Macedonia & 5.9849 & 0.0147 & 5.9781 & 0.0079 & 28.2280 & 0.0064 & 28.2486 & 0.0269 \\
\hline Madagascar & 32.1777 & -0.0019 & 32.1732 & -0.0064 & 40.0645 & -0.0034 & 40.0531 & -0.0148 \\
\hline Malawi & 7.4308 & 0.0124 & 7.5967 & 0.1783 & 3.1914 & 0.0796 & 3.4974 & 0.3856 \\
\hline Maldives & 0.0000 & 0.0000 & 0.0000 & 0.0000 & 0.0000 & 0.0000 & 0.0000 & 0.0000 \\
\hline Mali & 0.0000 & 0.0000 & 0.0000 & 0.0000 & 0.0000 & 0.0000 & 0.0000 & 0.0000 \\
\hline Malta & 0.0000 & 0.0000 & 0.0000 & 0.0000 & 8.3159 & 0.0085 & 8.3075 & 0.0000 \\
\hline Mauritius & 13.5227 & -0.0022 & 13.5250 & 0.0000 & 38.7434 & -0.0499 & 38.7932 & 0.0000 \\
\hline Mexico & 0.0000 & 0.0000 & 0.0000 & 0.0000 & 27.9968 & -0.0033 & 28.0264 & 0.0264 \\
\hline Moldova & 0.0000 & 0.0000 & 0.0000 & 0.0000 & 39.0884 & -0.0090 & 39.0082 & -0.0892 \\
\hline Mongolia & 6.3483 & -0.5413 & 6.8895 & 0.0000 & 0.0000 & 0.0000 & 0.0000 & 0.0000 \\
\hline Morocco & 0.0000 & 0.0000 & 0.0000 & 0.0000 & 0.0000 & 0.0000 & 0.0000 & 0.0000 \\
\hline Mozambique & 0.0000 & 0.0000 & 0.0000 & 0.0000 & 0.0000 & 0.0000 & 0.0000 & 0.0000 \\
\hline Namibia & 1.3388 & 0.2113 & 1.1275 & 0.0000 & 0.0000 & 0.0000 & 0.0000 & 0.0000 \\
\hline Nepal & 74.4329 & -0.0029 & 74.4375 & 0.0018 & 43.0448 & -0.0177 & 43.0762 & 0.0137 \\
\hline Netherlands & 0.0000 & 0.0000 & 0.0000 & 0.0000 & 38.1764 & -0.0090 & 38.1854 & 0.0000 \\
\hline Nicaragua & 52.3281 & 0.0040 & 52.3819 & 0.0578 & 24.3967 & 0.0258 & 24.4959 & 0.1250 \\
\hline
\end{tabular}


Table A2. Cont.

\begin{tabular}{|c|c|c|c|c|c|c|c|c|}
\hline \multirow{3}{*}{$\mathbf{D M U}_{j}$} & \multicolumn{4}{|c|}{ Methane Emissions $z_{1}^{b}$} & \multicolumn{4}{|c|}{ Nitrous Oxide Emissions $z_{2}^{b}$} \\
\hline & \multicolumn{2}{|c|}{ w.r.t $y_{1}$} & \multicolumn{2}{|c|}{$\begin{array}{c}1 \\
\text { w.r.t } y_{2}\end{array}$} & \multicolumn{2}{|c|}{ w.r.t $y_{1}$} & \multicolumn{2}{|c|}{ w.r.t $y_{2}$} \\
\hline & $z_{1}^{b+}$ & $\tau_{11}^{+}$ & $z_{1}^{b+}$ & $\tau_{12}^{+}$ & $z_{2}^{b+}$ & $\tau_{21}^{+}$ & $z_{2}^{b+}$ & $\tau_{22}^{+}$ \\
\hline Niger & 0.0000 & 0.0000 & 0.0000 & 0.0000 & 0.0000 & 0.0000 & 0.0000 & 0.0000 \\
\hline Nigeria & 0.0000 & 0.0000 & 0.0000 & 0.0000 & 0.0000 & 0.0000 & 0.0000 & 0.0000 \\
\hline Norway & 7.1694 & -0.0028 & 7.1756 & 0.0034 & 41.9804 & 0.0009 & 41.8580 & -0.1215 \\
\hline Oman & 4.4126 & -0.0003 & 4.4128 & 0.0000 & 26.1590 & -0.0063 & 26.1652 & 0.0000 \\
\hline Pakistan & 30.4262 & -0.0306 & 30.4568 & 0.0000 & 0.0000 & 0.0000 & 0.0000 & 0.0000 \\
\hline Panama & 19.0953 & 0.0933 & 18.8226 & -0.1794 & 0.0000 & 0.0000 & 0.0000 & 0.0000 \\
\hline $\begin{array}{l}\text { Papua New } \\
\text { Guinea }\end{array}$ & 0.0000 & 0.0000 & 0.0000 & 0.0000 & 0.0000 & 0.0000 & 0.0000 & 0.0000 \\
\hline Paraguay & 0.0000 & 0.0000 & 0.0000 & 0.0000 & 31.8761 & 0.0653 & 31.7728 & -0.0381 \\
\hline Philippines & 54.0918 & -0.0010 & 54.0928 & 0.0000 & 56.6386 & -0.0223 & 56.6609 & 0.0000 \\
\hline Poland & 21.0054 & -0.0013 & 21.0066 & 0.0000 & 34.4932 & -0.0280 & 34.5212 & 0.0000 \\
\hline Portugal & 26.1282 & -0.0013 & 26.1295 & 0.0000 & 38.1376 & -0.0294 & 38.1670 & 0.0000 \\
\hline Romania & 0.0000 & 0.0000 & 0.0000 & 0.0000 & 5.6961 & 0.0154 & 5.7570 & 0.0763 \\
\hline Rwanda & 0.0000 & 0.0000 & 0.0000 & 0.0000 & 11.4551 & 0.0261 & 11.5463 & 0.1173 \\
\hline Saudi Arabia & 0.0000 & 0.0000 & 0.0000 & 0.0000 & 18.3427 & -0.0295 & 18.3722 & 0.0000 \\
\hline Senegal & 31.8184 & 0.0691 & 31.9967 & 0.2475 & 0.0000 & 0.0000 & 0.0000 & 0.0000 \\
\hline Slovak Republic & 21.7400 & 0.0160 & 21.6718 & -0.0522 & 1.8577 & 0.0198 & 1.7255 & -0.1124 \\
\hline Slovenia & 26.3791 & 0.0000 & 26.3819 & 0.0028 & 35.5002 & 0.0000 & 35.5621 & 0.0619 \\
\hline South Africa & 0.0000 & 0.0000 & 0.0000 & 0.0000 & 0.0000 & 0.0000 & 0.0000 & 0.0000 \\
\hline Spain & 0.0000 & 0.0000 & 0.0000 & 0.0000 & 5.7476 & -0.0139 & 5.8735 & 0.1120 \\
\hline Sri Lanka & 55.5878 & -0.0005 & 55.5883 & 0.0000 & 33.7308 & -0.0115 & 33.7423 & 0.0000 \\
\hline St. Kitts and Nevis & 0.0000 & 0.0000 & 0.0000 & 0.0000 & 0.0000 & 0.0000 & 0.0000 & 0.0000 \\
\hline St. Lucia & 31.6428 & 0.0247 & 31.5377 & -0.0804 & 1.7324 & 0.0306 & 1.5287 & -0.1732 \\
\hline Suriname & 0.0000 & 0.0000 & 0.0000 & 0.0000 & 0.0000 & 0.0000 & 0.0000 & 0.0000 \\
\hline Sweden & 0.0000 & 0.0000 & 0.0000 & 0.0000 & 0.0000 & 0.0000 & 0.0000 & 0.0000 \\
\hline Switzerland & 57.7132 & -0.0027 & 57.7192 & 0.0033 & 41.0546 & 0.0009 & 40.9359 & -0.1179 \\
\hline Tajikistan & 0.0000 & 0.0000 & 0.0000 & 0.0000 & 0.0000 & 0.0000 & 0.0000 & 0.0000 \\
\hline Tanzania & 0.0000 & 0.0000 & 0.0000 & 0.0000 & 0.0000 & 0.0000 & 0.0000 & 0.0000 \\
\hline Thailand & 52.6556 & -0.0044 & 52.6708 & 0.0109 & 38.9072 & -0.0267 & 38.9528 & 0.0189 \\
\hline Togo & 0.0000 & 0.0000 & 0.0000 & 0.0000 & 0.0000 & 0.0000 & 0.0000 & 0.0000 \\
\hline Trinidad, Tobago & 0.0000 & 0.0000 & 0.0000 & 0.0000 & 0.0000 & 0.0000 & 0.0000 & 0.0000 \\
\hline Tunisia & 0.0000 & 0.0000 & 0.0000 & 0.0000 & 8.8994 & 0.0388 & 8.8606 & 0.0000 \\
\hline Turkey & 22.8057 & -0.0011 & 22.8067 & 0.0000 & 48.6553 & -0.0234 & 48.6787 & 0.0000 \\
\hline Uganda & 14.3203 & 0.0123 & 40.7055 & 26.3974 & 0.0000 & 0.0000 & 61.1962 & 61.1962 \\
\hline Ukraine & 0.0000 & 0.0000 & 0.0000 & 0.0000 & 0.0000 & 0.0000 & 0.0000 & 0.0000 \\
\hline UAE & 5.3534 & -0.0024 & 5.3531 & -0.0028 & 27.0438 & -0.0374 & 26.9642 & -0.1170 \\
\hline United Kingdom & 0.0000 & 0.0000 & 0.0000 & 0.0000 & 23.7600 & -0.0133 & 23.7733 & 0.0000 \\
\hline Uruguay & 0.0000 & 0.0000 & 0.0000 & 0.0000 & 0.0000 & 0.0000 & 0.0000 & 0.0000 \\
\hline Uzbekistan & 19.9724 & 0.0006 & 19.9717 & 0.0000 & 59.7668 & 0.0144 & 59.7524 & 0.0000 \\
\hline Venezuela, RB & 38.2000 & -0.0013 & 38.2028 & 0.0016 & 39.7525 & 0.0004 & 39.6965 & -0.0556 \\
\hline Vietnam & 52.2606 & -0.0003 & 52.2612 & 0.0003 & 52.7845 & 0.0001 & 52.7732 & -0.0112 \\
\hline Yemen, Rep. & 0.0000 & 0.0000 & 0.0000 & 0.0000 & 0.0000 & 0.0000 & 0.0000 & 0.0000 \\
\hline Zambia & 35.7680 & -0.0184 & 35.8049 & 0.0184 & 0.0000 & 0.0000 & 0.0000 & 0.0000 \\
\hline Zimbabwe & 35.3227 & 0.0079 & 35.3408 & 0.0259 & 27.0798 & 0.0138 & 27.1261 & 0.0601 \\
\hline
\end{tabular}

Table A3. Trade-off effects on the methane $\left(z_{1}^{b}\right)$ and nitrous oxide $\left(z_{2}^{b}\right)$ when $h=-0.5$ and $d=(1,1)$.

\begin{tabular}{|c|c|c|c|c|c|c|c|c|}
\hline \multirow{3}{*}{$\mathrm{DMU}_{j}$} & \multicolumn{4}{|c|}{ Methane Emissions $z_{1}^{b}$} & \multicolumn{4}{|c|}{ Nitrous Oxide Emissions $z_{2}^{b}$} \\
\hline & \multicolumn{2}{|c|}{ w.r.t $y_{1}$} & \multicolumn{2}{|c|}{ w.r.t $y_{2}$} & \multicolumn{2}{|c|}{ w.r.t $y_{1}$} & \multicolumn{2}{|c|}{ w.r.t $y_{2}$} \\
\hline & $z_{1}^{-}$ & $\tau_{11}^{-}$ & $z_{1}^{-}$ & $\tau_{12}^{-}$ & $z_{2}^{-}$ & $\tau_{21}^{-}$ & $z_{2}^{-}$ & $\tau_{22}^{-}$ \\
\hline Afghanistan & 11.3900 & -0.0071 & 11.3971 & 0.0000 & 0.0000 & 0.0000 & 0.0000 & 0.0000 \\
\hline Albania & 33.9745 & -0.1843 & 34.1588 & 0.0000 & 36.9676 & -0.1287 & 37.0963 & 0.0000 \\
\hline Algeria & 0.0000 & 0.0000 & 0.0000 & 0.0000 & 0.0000 & 0.0000 & 0.0000 & 0.0000 \\
\hline Angola & 0.0000 & 0.0000 & 0.0000 & 0.0000 & 0.0000 & 0.0000 & 0.0000 & 0.0000 \\
\hline
\end{tabular}


Table A3. Cont.

\begin{tabular}{|c|c|c|c|c|c|c|c|c|}
\hline \multirow{3}{*}{$\mathrm{DMU}_{j}$} & \multicolumn{4}{|c|}{ Methane Emissions $z_{1}^{b}$} & \multicolumn{4}{|c|}{ Nitrous Oxide Emissions $z_{2}^{b}$} \\
\hline & \multicolumn{2}{|c|}{ w.r.t $y_{1}$} & \multicolumn{2}{|c|}{ w.r.t $y_{2}$} & \multicolumn{2}{|c|}{ w.r.t $y_{1}$} & \multicolumn{2}{|c|}{ w.r.t $y_{2}$} \\
\hline & $z_{1}^{-}$ & $\tau_{11}^{-}$ & $z_{1}^{-}$ & $\tau_{12}^{-}$ & $z_{2}^{-}$ & $\tau_{21}^{-}$ & $z_{2}^{-}$ & $\tau_{22}^{-}$ \\
\hline Antigua, Barbuda & 0.0000 & 0.0000 & 0.0000 & 0.0000 & 0.0000 & 0.0000 & 0.0000 & 0.0000 \\
\hline Argentina & 0.0000 & 0.0000 & 0.0000 & 0.0000 & 0.0000 & 0.0000 & 0.0000 & 0.0000 \\
\hline Armenia & 0.0000 & 0.0000 & 0.0000 & 0.0000 & 16.4509 & -0.0586 & 16.5095 & 0.0000 \\
\hline Australia & 0.0000 & 0.0000 & 0.0000 & 0.0000 & 49.3314 & 0.0034 & 49.3280 & 0.0000 \\
\hline Austria & 45.9665 & 0.0018 & 45.9624 & -0.0023 & 38.4108 & -0.0006 & 38.4927 & 0.0812 \\
\hline Azerbaijan & 0.0000 & 0.0000 & 0.0000 & 0.0000 & 11.0384 & 0.0073 & 11.0310 & 0.0000 \\
\hline Bahamas, The & 0.0000 & 0.0000 & 0.0000 & 0.0000 & 0.0000 & 0.0000 & 0.0000 & 0.0000 \\
\hline Bangladesh & 62.1300 & -0.0001 & 62.1302 & 0.0000 & 54.2828 & -0.0028 & 54.2856 & 0.0000 \\
\hline Barbados & 0.0000 & 0.0000 & 0.0000 & 0.0000 & 8.4524 & 0.0219 & 8.7039 & 0.2734 \\
\hline Belarus & 43.2096 & -0.0124 & 43.2634 & 0.0414 & 40.7744 & -0.0154 & 40.8790 & 0.0892 \\
\hline Belgium & 43.5784 & -0.0589 & 43.6373 & 0.0000 & 0.0000 & 0.0000 & 0.0000 & 0.0000 \\
\hline Belize & 51.0052 & 0.0000 & 51.0033 & -0.0020 & 33.9164 & 0.0000 & 33.8727 & -0.0437 \\
\hline Benin & 14.2538 & -0.0664 & 14.0673 & -0.2528 & 0.0000 & 0.0000 & 0.0000 & 0.0000 \\
\hline Bhutan & 0.0000 & 0.0000 & 0.0000 & 0.0000 & 0.0000 & 0.0000 & 0.0000 & 0.0000 \\
\hline Bolivia & 20.7541 & 0.0007 & 20.7549 & 0.0015 & 15.4104 & -0.0005 & 15.4113 & 0.0004 \\
\hline $\begin{array}{l}\text { Bosnia, } \\
\text { Herzegovina }\end{array}$ & 34.9269 & -0.0122 & 34.9798 & 0.0407 & 62.5537 & -0.0151 & 62.6565 & 0.0877 \\
\hline Botswana & 0.0000 & 0.0000 & 0.0000 & 0.0000 & 46.6598 & -0.0158 & 46.6756 & 0.0000 \\
\hline Brunei Darussalam & 0.0000 & 0.0000 & 0.0000 & 0.0000 & 0.0000 & 0.0000 & 0.0000 & 0.0000 \\
\hline Bulgaria & 7.0051 & 0.0009 & 7.0042 & 0.0000 & 31.7221 & 0.0210 & 31.7010 & 0.0000 \\
\hline Burkina Faso & 0.0000 & 0.0000 & 0.0000 & 0.0000 & 0.0000 & 0.0000 & 0.0000 & 0.0000 \\
\hline Burundi & 0.0000 & 0.0000 & 0.0000 & 0.0000 & 0.0000 & 0.0000 & 0.0000 & 0.0000 \\
\hline Cambodia & 0.0000 & 0.0000 & 0.0000 & 0.0000 & 0.0000 & 0.0000 & 0.0000 & 0.0000 \\
\hline Cameroon & 0.0000 & 0.0000 & 0.0000 & 0.0000 & 0.0000 & 0.0000 & 0.0000 & 0.0000 \\
\hline $\begin{array}{l}\text { Central African } \\
\text { Rep }\end{array}$ & 0.0000 & 0.0000 & 0.0000 & 0.0000 & 0.0000 & 0.0000 & 0.0000 & 0.0000 \\
\hline Chile & 33.6993 & 0.0011 & 33.6982 & 0.0000 & 35.5376 & 0.0253 & 35.5122 & 0.0000 \\
\hline Congo, Dem. Rep. & 0.0000 & 0.0000 & 0.0000 & 0.0000 & 0.0000 & 0.0000 & 0.0000 & 0.0000 \\
\hline Congo, Rep. & 0.0000 & 0.0000 & 0.0000 & 0.0000 & 0.0000 & 0.0000 & 0.0000 & 0.0000 \\
\hline Costa Rica & 61.9984 & 0.0001 & 61.9981 & -0.0001 & 45.8673 & 0.0000 & 45.8726 & 0.0053 \\
\hline Cote d'Ivoire & 12.0193 & 0.0043 & 12.0124 & -0.0027 & 3.4958 & 0.0262 & 3.4495 & -0.0202 \\
\hline Croatia & 16.7488 & 0.0008 & 16.7480 & 0.0000 & 26.9355 & 0.0177 & 26.9179 & 0.0000 \\
\hline Cuba & 5.4733 & -0.0241 & 5.4802 & -0.0173 & 16.9674 & -0.0095 & 16.9217 & -0.0552 \\
\hline Cyprus & 40.0264 & 0.0000 & 40.0351 & 0.0087 & 42.0092 & 0.0000 & 42.2018 & 0.1926 \\
\hline Czech Republic & 28.4080 & 0.0013 & 28.4067 & 0.0000 & 65.3085 & 0.0286 & 65.2799 & 0.0000 \\
\hline Denmark & 0.0000 & 0.0000 & 0.0000 & 0.0000 & 58.9398 & 0.0029 & 58.9369 & 0.0000 \\
\hline Dominica & 0.0000 & 0.0000 & 0.0000 & 0.0000 & 0.0000 & 0.0000 & 0.0000 & 0.0000 \\
\hline $\begin{array}{l}\text { Dominican } \\
\text { Republic }\end{array}$ & 0.0000 & 0.0000 & 0.0000 & 0.0000 & 18.3667 & -0.0405 & 18.4072 & 0.0000 \\
\hline Ecuador & 64.6133 & 0.0010 & 64.6111 & -0.0012 & 54.8172 & -0.0003 & 54.8602 & 0.0427 \\
\hline Egypt, Arab Rep. & 26.8428 & 0.0009 & 26.8419 & 0.0000 & 41.7122 & 0.0198 & 41.6924 & 0.0000 \\
\hline El Salvador & 0.0000 & 0.0000 & 0.0000 & 0.0000 & 30.9846 & 0.0044 & 30.9802 & 0.0000 \\
\hline Eritrea & 28.1384 & -0.0090 & 28.1474 & 0.0000 & 0.0000 & 0.0000 & 0.0000 & 0.0000 \\
\hline Estonia & 0.0000 & 0.0000 & 0.0000 & 0.0000 & 0.0000 & 0.0000 & 0.0000 & 0.0000 \\
\hline Ethiopia & 0.0000 & 0.0000 & 0.0000 & 0.0000 & 24.9627 & 10.4356 & 14.5271 & 0.0000 \\
\hline Fiji & 52.9614 & 0.0077 & 52.9291 & -0.0245 & 21.6796 & -0.0134 & 21.6349 & -0.0581 \\
\hline Finland & 0.0000 & 0.0000 & 0.0000 & 0.0000 & 0.0000 & 0.0000 & 0.0000 & 0.0000 \\
\hline France & 0.0000 & 0.0000 & 0.0000 & 0.0000 & 39.4887 & -0.0007 & 39.5241 & 0.0347 \\
\hline Gabon & 0.0000 & 0.0000 & 0.0000 & 0.0000 & 0.0000 & 0.0000 & 0.0000 & 0.0000 \\
\hline Gambia, The & 12.8221 & -0.0369 & 12.7357 & -0.1233 & 0.3101 & -0.0645 & 0.0887 & -0.2859 \\
\hline Georgia & 40.2571 & 0.0000 & 40.2599 & 0.0028 & 25.5848 & 0.0000 & 25.6461 & 0.0613 \\
\hline Germany & 0.0000 & 0.0000 & 0.0000 & 0.0000 & 10.6897 & 0.0208 & 10.6618 & -0.0072 \\
\hline Ghana & 7.1463 & -0.0142 & 6.9528 & -0.2077 & 4.6209 & -0.0911 & 4.2629 & -0.4492 \\
\hline Greece & 32.6142 & -0.0321 & 32.7537 & 0.1074 & 41.4223 & -0.0398 & 41.6934 & 0.2313 \\
\hline Guatemala & 47.2302 & -0.0007 & 47.2309 & 0.0000 & 24.9869 & -0.0154 & 25.0023 & 0.0000 \\
\hline
\end{tabular}


Table A3. Cont.

\begin{tabular}{|c|c|c|c|c|c|c|c|c|}
\hline \multirow{3}{*}{$\mathrm{DMU}_{j}$} & \multicolumn{4}{|c|}{ Methane Emissions $z_{1}^{b}$} & \multicolumn{4}{|c|}{ Nitrous Oxide Emissions $z_{2}^{b}$} \\
\hline & \multicolumn{2}{|c|}{ w.r.t $y_{1}$} & \multicolumn{2}{|c|}{ w.r.t $y_{2}$} & \multicolumn{2}{|c|}{ w.r.t $y_{1}$} & \multicolumn{2}{|c|}{ w.r.t $y_{2}$} \\
\hline & $z_{1}^{-}$ & $\tau_{11}^{-}$ & $z_{1}^{-}$ & $\tau_{12}^{-}$ & $z_{2}^{-}$ & $\tau_{21}^{-}$ & $z_{2}^{-}$ & $\tau_{22}^{-}$ \\
\hline Guinea & 18.7279 & -0.0029 & 18.7129 & -0.0179 & 0.0000 & 0.0000 & 0.0000 & 0.0000 \\
\hline Guyana & 0.0000 & 0.0000 & 0.0000 & 0.0000 & 0.0000 & 0.0000 & 0.0000 & 0.0000 \\
\hline Honduras & 72.4088 & 0.0004 & 72.4084 & 0.0000 & 41.7889 & 0.0094 & 41.7795 & 0.0000 \\
\hline Hungary & 0.0000 & 0.0000 & 0.0000 & 0.0000 & 88.6828 & 0.0311 & 88.6517 & 0.0000 \\
\hline Iceland & 54.8234 & 0.0000 & 54.8234 & 0.0000 & 43.6820 & 0.0018 & 43.6802 & 0.0000 \\
\hline Iran, Islamic Rep. & 17.4558 & 0.0078 & 0.0000 & -17.4480 & 57.4069 & 0.0443 & 19.6190 & -37.7437 \\
\hline Iraq & 0.0000 & 0.0000 & 0.0000 & 0.0000 & 0.0000 & 0.0000 & 0.0000 & 0.0000 \\
\hline Israel & 0.0000 & 0.0000 & 0.0000 & 0.0000 & 28.9523 & 0.0199 & 28.9324 & 0.0000 \\
\hline Italy & 0.0000 & 0.0000 & 0.0000 & 0.0000 & 35.6976 & -0.0042 & 35.7364 & 0.0346 \\
\hline Jamaica & 3.9417 & 0.0025 & 3.9190 & -0.0203 & 0.0000 & 0.0000 & 0.0000 & 0.0000 \\
\hline Japan & 0.0000 & 0.0000 & 0.0000 & 0.0000 & 0.0000 & 0.0000 & 0.0000 & 0.0000 \\
\hline Jordan & 0.0000 & 0.0000 & 0.0000 & 0.0000 & 44.6324 & -0.0005 & 44.6329 & 0.0000 \\
\hline Kazakhstan & 0.0000 & 0.0000 & 0.0000 & 0.0000 & 0.0000 & 0.0000 & 0.0000 & 0.0000 \\
\hline Kenya & 0.0000 & 0.0000 & 0.0000 & 0.0000 & 0.0000 & 0.0000 & 0.0000 & 0.0000 \\
\hline Korea, Rep. & 33.3122 & 0.0007 & 33.3106 & -0.0009 & 42.2033 & -0.0002 & 42.2340 & 0.0305 \\
\hline Kuwait & 0.0000 & 0.0000 & 0.0000 & 0.0000 & 0.0000 & 0.0000 & 0.0000 & 0.0000 \\
\hline Kyrgyz Republic & 0.0000 & 0.0000 & 0.0000 & 0.0000 & 0.0000 & 0.0000 & 0.0000 & 0.0000 \\
\hline Latvia & 0.5596 & -0.0151 & 0.4502 & -0.1245 & 34.1123 & -0.0690 & 33.9119 & -0.2694 \\
\hline Lebanon & 25.1225 & 0.0018 & 25.1207 & 0.0000 & 64.8823 & 0.0408 & 64.8415 & 0.0000 \\
\hline Lithuania & 0.0000 & 0.0000 & 0.0000 & 0.0000 & 0.0000 & 0.0000 & 0.0000 & 0.0000 \\
\hline Luxembourg & 30.2075 & -0.0369 & 30.2180 & -0.0264 & 1.1175 & -0.0145 & 1.0476 & -0.0844 \\
\hline North Macedonia & 5.9554 & -0.0148 & 5.9622 & -0.0081 & 28.2153 & -0.0064 & 28.1942 & -0.0275 \\
\hline Madagascar & 32.1815 & 0.0019 & 32.1861 & 0.0065 & 40.0712 & 0.0034 & 40.0829 & 0.0151 \\
\hline Malawi & 7.4059 & -0.0125 & 7.2361 & -0.1823 & 3.0319 & -0.0799 & 2.7177 & -0.3941 \\
\hline Maldives & 0.0000 & 0.0000 & 0.0000 & 0.0000 & 0.0000 & 0.0000 & 0.0000 & 0.0000 \\
\hline Mali & 0.0000 & 0.0000 & 0.0000 & 0.0000 & 0.0000 & 0.0000 & 0.0000 & 0.0000 \\
\hline Malta & 0.0000 & 0.0000 & 0.0000 & 0.0000 & 8.2990 & -0.0085 & 8.3075 & 0.0000 \\
\hline Mauritius & 13.5272 & 0.0023 & 13.5250 & 0.0000 & 38.8434 & 0.0502 & 38.7932 & 0.0000 \\
\hline Mexico & 0.0000 & 0.0000 & 0.0000 & 0.0000 & 28.0033 & 0.0033 & 27.9731 & -0.0269 \\
\hline Moldova & 0.0000 & 0.0000 & 0.0000 & 0.0000 & 39.1064 & 0.0090 & 39.1879 & 0.0905 \\
\hline Mongolia & 7.4302 & 0.5406 & 6.8895 & 0.0000 & 0.0000 & 0.0000 & 0.0000 & 0.0000 \\
\hline Morocco & 0.0000 & 0.0000 & 0.0000 & 0.0000 & 0.0000 & 0.0000 & 0.0000 & 0.0000 \\
\hline Mozambique & 0.0000 & 0.0000 & 0.0000 & 0.0000 & 0.0000 & 0.0000 & 0.0000 & 0.0000 \\
\hline Namibia & 0.9161 & -0.2115 & 1.1275 & 0.0000 & 0.0000 & 0.0000 & 0.0000 & 0.0000 \\
\hline Nepal & 74.4386 & 0.0029 & 74.4339 & -0.0018 & 43.0803 & 0.0178 & 43.0488 & -0.0137 \\
\hline Netherlands & 0.0000 & 0.0000 & 0.0000 & 0.0000 & 38.1945 & 0.0091 & 38.1854 & 0.0000 \\
\hline Nicaragua & 52.3201 & -0.0040 & 52.2650 & -0.0591 & 24.3450 & -0.0259 & 24.2431 & -0.1278 \\
\hline Niger & 0.0000 & 0.0000 & 0.0000 & 0.0000 & 0.0000 & 0.0000 & 0.0000 & 0.0000 \\
\hline Nigeria & 0.0000 & 0.0000 & 0.0000 & 0.0000 & 0.0000 & 0.0000 & 0.0000 & 0.0000 \\
\hline Norway & 7.1749 & 0.0028 & 7.1687 & -0.0035 & 41.9786 & -0.0009 & 42.1022 & 0.1227 \\
\hline Oman & 4.4131 & 0.0003 & 4.4128 & 0.0000 & 26.1715 & 0.0063 & 26.1652 & 0.0000 \\
\hline Pakistan & 30.4875 & 0.0307 & 30.4568 & 0.0000 & 0.0000 & 0.0000 & 0.0000 & 0.0000 \\
\hline Panama & 18.9085 & -0.0935 & 19.1822 & 0.1802 & 0.0000 & 0.0000 & 0.0000 & 0.0000 \\
\hline $\begin{array}{l}\text { Papua New } \\
\text { Guinea }\end{array}$ & 0.0000 & 0.0000 & 0.0000 & 0.0000 & 0.0000 & 0.0000 & 0.0000 & 0.0000 \\
\hline Paraguay & 0.0000 & 0.0000 & 0.0000 & 0.0000 & 31.7452 & -0.0656 & 31.8491 & 0.0382 \\
\hline Philippines & 54.0938 & 0.0010 & 54.0928 & 0.0000 & 56.6833 & 0.0225 & 56.6609 & 0.0000 \\
\hline Poland & 21.0079 & 0.0013 & 21.0066 & 0.0000 & 34.5494 & 0.0282 & 34.5212 & 0.0000 \\
\hline Portugal & 26.1308 & 0.0013 & 26.1295 & 0.0000 & 38.1966 & 0.0296 & 38.1670 & 0.0000 \\
\hline Romania & 0.0000 & 0.0000 & 0.0000 & 0.0000 & 5.6652 & -0.0155 & 5.6028 & -0.0780 \\
\hline Rwanda & 0.0000 & 0.0000 & 0.0000 & 0.0000 & 11.4028 & -0.0262 & 11.3085 & -0.1206 \\
\hline Saudi Arabia & 0.0000 & 0.0000 & 5.3588 & 5.3588 & 18.4018 & 0.0296 & 29.9140 & 11.5419 \\
\hline Senegal & 31.6801 & -0.0692 & 31.5000 & -0.2492 & 0.0000 & 0.0000 & 0.0000 & 0.0000 \\
\hline Slovak Republic & 21.7080 & -0.0160 & 21.7774 & 0.0535 & 1.8180 & -0.0198 & 1.9530 & 0.1151 \\
\hline Slovenia & 26.3791 & 0.0000 & 26.3763 & -0.0028 & 35.5002 & 0.0000 & 35.4371 & -0.0631 \\
\hline
\end{tabular}


Table A3. Cont.

\begin{tabular}{|c|c|c|c|c|c|c|c|c|}
\hline \multirow{3}{*}{$\mathrm{DMU}_{j}$} & \multicolumn{4}{|c|}{ Methane Emissions $z_{1}^{b}$} & \multicolumn{4}{|c|}{ Nitrous Oxide Emissions $z_{2}^{b}$} \\
\hline & \multicolumn{2}{|c|}{ w.r.t $y_{1}$} & \multicolumn{2}{|c|}{ w.r.t $y_{2}$} & \multicolumn{2}{|c|}{ w.r.t $y_{1}$} & \multicolumn{2}{|c|}{ w.r.t $y_{2}$} \\
\hline & $z_{1}^{-}$ & $\tau_{11}^{-}$ & $z_{1}^{-}$ & $\tau_{12}^{-}$ & $z_{2}^{-}$ & $\tau_{21}^{-}$ & $z_{2}^{-}$ & $\tau_{22}^{-}$ \\
\hline South Africa & 0.0000 & 0.0000 & 0.0000 & 0.0000 & 0.0000 & 0.0000 & 0.0000 & 0.0000 \\
\hline Spain & 0.0000 & 0.0000 & 0.0000 & 0.0000 & 5.7754 & 0.0139 & 5.6474 & -0.1141 \\
\hline Sri Lanka & 55.5888 & 0.0005 & 55.5883 & 0.0000 & 33.7539 & 0.0116 & 33.7423 & 0.0000 \\
\hline St. Kitts and Nevis & 0.0000 & 0.0000 & 0.0000 & 0.0000 & 0.0000 & 0.0000 & 0.0000 & 0.0000 \\
\hline St. Lucia & 31.5935 & -0.0246 & 31.7005 & 0.0824 & 1.6713 & -0.0305 & 1.8793 & 0.1774 \\
\hline Suriname & 0.0000 & 0.0000 & 0.0000 & 0.0000 & 0.0000 & 0.0000 & 0.0000 & 0.0000 \\
\hline Sweden & 0.0000 & 0.0000 & 0.0000 & 0.0000 & 0.0000 & 0.0000 & 0.0000 & 0.0000 \\
\hline Switzerland & 57.7186 & 0.0027 & 57.7125 & -0.0034 & 41.0528 & -0.0009 & 41.1728 & 0.1191 \\
\hline Tajikistan & 0.0000 & 0.0000 & 0.0000 & 0.0000 & 0.0000 & 0.0000 & 0.0000 & 0.0000 \\
\hline Tanzania & 0.0000 & 0.0000 & 0.0000 & 0.0000 & 0.0000 & 0.0000 & 0.0000 & 0.0000 \\
\hline Thailand & 52.6643 & 0.0044 & 52.6490 & -0.0109 & 38.9607 & 0.0268 & 38.9149 & -0.0190 \\
\hline Togo & 0.0000 & 0.0000 & 0.0000 & 0.0000 & 0.0000 & 0.0000 & 0.0000 & 0.0000 \\
\hline $\begin{array}{l}\text { Trinidad and } \\
\text { Tobago }\end{array}$ & 0.0000 & 0.0000 & 0.0000 & 0.0000 & 0.0000 & 0.0000 & 0.0000 & 0.0000 \\
\hline Tunisia & 0.0000 & 0.0000 & 0.0000 & 0.0000 & 8.8217 & -0.0389 & 8.8606 & 0.0000 \\
\hline Turkey & 22.8078 & 0.0011 & 22.8067 & 0.0000 & 48.7023 & 0.0235 & 48.6787 & 0.0000 \\
\hline Uganda & 14.2958 & -0.0123 & 14.3081 & 0.0000 & 0.0000 & 0.0000 & 0.0000 & 0.0000 \\
\hline Ukraine & 0.0000 & 0.0000 & 0.0000 & 0.0000 & 0.0000 & 0.0000 & 0.0000 & 0.0000 \\
\hline $\begin{array}{l}\text { United Arab } \\
\text { Emirates }\end{array}$ & 5.3583 & 0.0025 & 5.3589 & 0.0030 & 27.1187 & 0.0376 & 27.2035 & 0.1223 \\
\hline United Kingdom & 0.0000 & 0.0000 & 0.0000 & 0.0000 & 23.7867 & 0.0134 & 23.7733 & 0.0000 \\
\hline Uruguay & 0.0000 & 0.0000 & 0.0000 & 0.0000 & 0.0000 & 0.0000 & 0.0000 & 0.0000 \\
\hline Uzbekistan & 19.9711 & -0.0006 & 0.0000 & -19.9717 & 59.7380 & -0.0145 & 16.7369 & -43.0156 \\
\hline Venezuela, RB & 38.2025 & 0.0013 & 38.1997 & -0.0016 & 39.7516 & -0.0004 & 39.8082 & 0.0562 \\
\hline Vietnam & 52.2611 & 0.0003 & 52.2606 & -0.0003 & 52.7843 & -0.0001 & 52.7957 & 0.0113 \\
\hline Yemen, Rep. & 0.0000 & 0.0000 & 0.0000 & 0.0000 & 0.0000 & 0.0000 & 0.0000 & 0.0000 \\
\hline Zambia & 35.8049 & 0.0184 & 35.7679 & -0.0186 & 0.0000 & 0.0000 & 0.0000 & 0.0000 \\
\hline Zimbabwe & 35.3069 & -0.0079 & 35.2883 & -0.0265 & 27.0521 & -0.0139 & 27.0044 & -0.0615 \\
\hline
\end{tabular}

\section{References}

1. Ivanic, M.; Martin, W. Sectoral productivity growth and poverty reduction: National and global impacts. World Dev. 2018, 109, 429-439. [CrossRef]

2. Picazo-Tadeo, A.J.; Gómez-Limón, J.A.; Reig-Martínez, E. Assessing farming eco-efficiency: A data envelopment analysis approach. J. Environ. Manag. 2011, 92, 1154-1164. [CrossRef] [PubMed]

3. Liu, L.-C.; Wu, G. The effects of carbon dioxide, methane and nitrous oxide emission taxes: An empirical study in China. J. Clean. Prod. 2017, 142, 1044-1054. [CrossRef]

4. Le Quéré, C.; Andrew, R.; Canadell, J.G.; Sitch, S.; Korsbakken, J.I.; Peters, G.P.; Manning, A.C.; Boden, T.A.; Tans, P.P.; Houghton, R.A. Global carbon budget 2016. Earth Syst. Sci. Data 2016, 8, 605-649. [CrossRef]

5. Klapwijk, C.J.; Van Wijk, M.T.; Rosenstock, T.S.; van Asten, P.J.A.; Thornton, P.K.; Giller, K.E. Analysis of trade-offs in agricultural systems: Current status and way forward. Curr. Opin. Environ. Sustain. 2014, 6, 110-115. [CrossRef]

6. Ndiaye, M.; Adam, M.; Ganyo, K.K.; Guissé, A.; Cissé, N.; Muller, B. Genotype-environment interaction: Trade-offs between the agronomic performance and stability of dual-purpose sorghum (Sorghum bicolor L. Moench) genotypes in Senegal. Agronomy 2019, 9, 867. [CrossRef]

7. Kanter, D.R.; Musumba, M.; Wood, S.L.R.; Palm, C.; Antle, J.; Balvanera, P.; Dale, V.H.; Havlik, P.; Kline, K.L.; Scholes, R.J. Evaluating agricultural trade-offs in the age of sustainable development. Agric. Syst. 2018, 163, 73-88. [CrossRef]

8. Da Silveira, G.; Slack, N. Exploring the trade-off concept. Int. J. Oper. Prod. Manag. 2001, 21, 949-964. [CrossRef]

9. Akbar, U.; Fan, R.N.; Li, Q.L. A route option for different commodity groups in international trade: China Pakistan economic corridor. In Communications in Computer and Information Science; Springer Nature: Singapore, 2019; Volume 1102, pp. 446-464.

10. Mirzaei, M.; Kordrostami, S.; Amirteimoori, A.; Chegini, M.G. Alternative trade-offs in data envelopment analysis: An application to hydropower plants. Math. Probl. Eng. 2016, 2016, 1-8. [CrossRef]

11. Khoshandam, L.; Amirteimoori, A.; Matin, R.K. Marginal rates of substitution in the presence of non-discretionary factors: A data envelopment analysis approach. Measurement 2014, 58, 409-415. [CrossRef]

12. Atici, K.B.; Podinovski, V. V Using data envelopment analysis for the assessment of technical efficiency of units with different specialisations: An application to agriculture. Omega 2015, 54, 72-83. [CrossRef]

13. Asmild, M.; Paradi, J.C.; Reese, D.N. Theoretical perspectives of trade-off analysis using DEA. Omega 2006, 34, 337-343. [CrossRef] 
14. Krivonozhko, V.E.; Utkin, O.B.; Volodin, A.V.; Sablin, I.A.; Patrin, M. Constructions of economic functions and calculations of marginal rates in DEA using parametric optimization methods. J. Oper. Res. Soc. 2004, 55, 1049-1058. [CrossRef]

15. Loizou, E.; Karelakis, C.; Galanopoulos, K.; Mattas, K. The role of agriculture as a development tool for a regional economy. Agric. Syst. 2019, 173, 482-490. [CrossRef]

16. Oláh, J.; Aburumman, N.; Popp, J.; Khan, M.A.; Haddad, H.; Kitukutha, N. Impact of Industry 4.0 on environmental sustainability. Sustainability 2020, 12, 4674.

17. Gružauskas, V.; Baskutis, S.; Navickas, V. Minimizing the trade-off between sustainability and cost effective performance by using autonomous vehicles. J. Clean. Prod. 2018, 184, 709-717. [CrossRef]

18. Shahbazpour, M.; Seidel, R. Strategic manufacturing system and process innovation through elimination of trade-offs. Int. J. Comput. Integr. Manuf. 2007, 20, 413-422. [CrossRef]

19. Pratt, C.; Kingston, K.; Laycock, B.; Levett, I.; Pratt, S. Geo-agriculture: Reviewing opportunities through which the geosphere can help address emerging crop production challenges. Agronomy 2020, 10, 971. [CrossRef]

20. Akbar, U.; Li, Q.L.; Akmal, M.A.; Shakib, M.; Iqbal, W. Nexus between agro-ecological efficiency and carbon emission transfer: Evidence from China. Environ. Sci. Pollut. Res. Int. 2020. [CrossRef]

21. Bosco, S.; Volpi, I.; Antichi, D.; Ragaglini, G.; Frasconi, C. Greenhouse gas emissions from soil cultivated with vegetables in crop rotation under Integrated, organic and organic conservation management in a mediterranean environment. Agronomy 2019, 9, 446. [CrossRef]

22. Shen, J.; Melaku, N.D.; Treu, R.; Wang, J. Inventories of methane and nitrous oxide emissions from animal and crop farms of 69 municipalities in Alberta, Canada. J. Clean. Prod. 2019, 234, 895-911. [CrossRef]

23. Yue, Q.; Wu, H.; Sun, J.; Cheng, K.; Smith, P.; Hillier, J.; Xu, X.; Pan, G. Deriving emission factors and estimating direct nitrous oxide emissions for crop cultivation in China. Environ. Sci. Technol. 2019, 53, 10246-10257. [CrossRef] [PubMed]

24. Tang, H.-M.; Xiao, X.-P.; Wang, K.; Li, W.-Y.; Liu, J.; Sun, J.-M. Methane and nitrous oxide emissions as affected by long-term fertilizer management from double-cropping paddy fields in Southern China. J. Agric. Sci. 2016, 154, 1378-1391. [CrossRef]

25. Audet, J.; Bastviken, D.; Bundschuh, M.; Buffam, I.; Feckler, A.; Klemedtsson, L.; Laudon, H.; Löfgren, S.; Natchimuthu, S.; Öquist, M. Forest streams are important sources for nitrous oxide emissions. Glob. Chang. Biol. 2020, 26, 629-641. [CrossRef]

26. Wysocka-Czubaszek, A.; Czubaszek, R.; Roj-Rojewski, S.; Banaszuk, P. Methane and nitrous oxide emissions from agriculture on a regional scale. J. Ecol. Eng. 2018, 19, 206-217. [CrossRef]

27. Wysocka-Czubaszek, A.; Banaszuk, P.; Czubaszek, R. Methane and nitrous oxide emissions from agriculture in the Podlaskie voivodeship in years 1999-2015. Pol. J. Nat. Sci 2018, 33, 433-453.

28. Hama-Aziz, Z.Q.; Hiscock, K.M.; Cooper, R.J. Indirect nitrous oxide emission factors for agricultural field drains and headwater streams. Environ. Sci. Technol. 2017, 51, 301-307. [CrossRef]

29. Rosen, D.; Schaffnit, C.; Paradi, J.C. Marginal rates and two-dimensional level curves in DEA. J. Product. Anal. 1998, 9, 205-232. [CrossRef]

30. Sueyoshi, T.; Goto, M. Returns to scale, damages to scale, marginal rate of transformation and rate of substitution in DEA environmental assessment. Energy Econ. 2012, 34, 905-917. [CrossRef]

31. Huang, Z.; Li, S.X.; Rousseau, J.J. Determining rates of change in data envelopment analysis. J. Oper. Res. Soc. 1997, 48, 591-599. [CrossRef]

32. Emrouznejad, A.; Yang, G. A survey and analysis of the first 40 years of scholarly literature in DEA: 1978-2016. Socioecon. Plann. Sci. 2018, 61, 4-8. [CrossRef]

33. Cooper, W.W.; Park, K.S.; Pastor Ciurana, J.T. Marginal rates and elasticities of substitution with additive models in DEA. J. Product. Anal. 2000, 13, 105-123. [CrossRef]

34. Chang, Y.T.; Zhang, N.; Danao, D.; Zhang, N. Environmental efficiency analysis of transportation system in China: A non-radial DEA approach. Energy Policy 2013, 58, 277-283. [CrossRef]

35. Watto, M.A.; Mugera, A.W. Irrigation water demand and implications for groundwater pricing in Pakistan. Water Policy 2016, 18, 565-585. [CrossRef]

36. Miller, N.J.; Bergtold, J.S.; Featherstone, A.M. Economic elasticities of input substitution using data envelopment analysis. PLoS ONE 2019, 14, e0220478. [CrossRef] [PubMed]

37. Akbar, U.; Kumar, A.; Khan, H.; Khan, M.A.; Parvaiz, K.; Oláh, J. Trade-offs in competitive transport operations. Economies 2020, 8, 56. [CrossRef]

38. Tone, K. Slacks-based measure of efficiency in data envelopment analysis. Eur. J. Oper. Res. 2001, 130, 498-509. [CrossRef]

39. Seiford, L.M.; Zhu, J. Modeling undesirable factors in efficiency evaluation. Eur. J. Oper. Res. 2002, 142, 16-20. [CrossRef]

40. Cooper, W.W.; Seiford, L.M.; Tone, K. Data Envelopment Analysis: A Comprehensive Text with Models, Applications, References and DEA-Solver Software, 2nd ed.; Springer: New York, NY, USA, 2007; ISBN 0387452818.

41. Shephard, R.W. Theory of Cost and Production Functions; Princeton University Press: Princeton, NJ, USA, 2015 ; ISBN 9781400871087.

42. Akbar, U.; Khan, M.A.; Akmal, M.; Tóth Naárné, É.Z.; Oláh, J. Trade-offs for the optimal energy efficiency of road transportation: Domestic cases in developing countries. Energies 2020, 13, 6538. [CrossRef]

43. Akbar, U.; Popp, J.; Khan, H.; Khan, M.A.; Oláh, J. Energy efficiency in transportation along with the belt and road countries. Energies 2020, 13, 2607. [CrossRef] 